\title{
A Geometric Model of Ion Orbit Loss Under the Influence of a Radial Electric Field
}

\author{
Robert W. Brzozowski III, ${ }^{1,2}$ Frank Jenko, ${ }^{2}$ Marco Cavedon, ${ }^{2}$ and the ASDEX Upgrade team ${ }^{2}$ \\ ${ }^{1)}$ Department of Physics and Astronomy, University of California, Los Angeles, 475 Portola Plaza, Los Angeles, \\ CA 90095-154\%, USA \\ ${ }^{2)}$ Max Planck Institute for Plasma Physics, Boltzmannstr. 2, 85748 Garching, \\ Germany
}

A geometric approach is used to find the threshold energies for ion orbit loss in a realistic tokamak geometry, like that of ASDEX Upgrade. The effects of any pre-existing radial electric field are maintained as they non-trivially affect the calculations. The associated velocity-space loss hole is investigated using equilibria and profiles from ASDEX Upgrade discharges 30628 and 31533. For the former, we consider the L-mode just before the L-H transition and also the resultant $\mathrm{H}$-mode. A single instance of the comparably high ion temperature H-mode of shot 31533 is investigated. We follow two ion orbit loss definitions, SOL sampling orbits and X-loss, and in both cases thermal ion orbit losses are significant in the pedestal region and scale with the ion temperature. The radial electric field plays a significant role in determining the orbits of ions, and consequentially swaths of configuration-space near the inner-midplane support toroidally trapped orbits under its influence. The accessibility of banana orbits is often dependent on an ion's energy which results in upwardly bounded velocity-space loss holes hereby called banana loss islands (BLIs). BLIs are prone to thermally refill under relaxation and should thus be considered when fully characterizing ion orbit loss.

\section{Introduction}

For a tokamak plasma, there is a regime of improved confinement that is achieved under the correct operating conditions ${ }^{1}$, the primary requirement being sufficient heating. This high confinement mode of operation, called the H-mode, in contrast to the lower confinement L-mode, is further associated with steep gradients in the density and temperature profiles near the plasma edge in a region frequently referred to as the plasma pedestal. These features are accompanied by a similarly strong radial electric field which provides for a local shear $E \times B$ poloidal flow thought to suppress local turbulent transport processes ${ }^{2,3}$. Thereupon, a connection between these characteristics and an edge transport barrier is made. For this reason, the $\mathrm{H}$-mode is commonly viewed as a necessary running condition for any efficient tokamak of the future, like ITER. This necessity has inspired much research into the physics of the plasma pedestal and its formation, the L-H transition.

Ion orbit loss has been studied as a potential cause, or as a contributing factor, for the $\mathrm{L}-\mathrm{H}$ transition following the discovery of the $\mathrm{H}$-mode $\mathrm{e}^{4,5}$ and is still an active research topic ${ }^{6-10}$. Between then and now, there has been a strong effort to understand ion orbit loss both analytically and computationally ${ }^{11-17}$. These efforts have shown that the axi-symmetric field geometry of a tokamak allows for the loss of thermal ions with orbits near the last closed flux surface (LCFS). The losses are non-ambipolar, since only the ions are affected, and are thus a potential source of the radial electric field in the edge. The magnitude of ion orbit loss, and the electric field thereby generated, shares sensitivities to both the $\nabla B$ drift direction and the $\mathrm{X}$-point geometry with the $\mathrm{L}-\mathrm{H}$ transition power thresholds ${ }^{5,17,18}$. Additionally, the heating condition necessary for the $\mathrm{L}-\mathrm{H}$ transition is possibly re- flected by the direct dependence of thermal ion orbit loss on the local ion temperature ${ }^{19}$. The typical methods of heating a tokamak, as well as the desired fusion events themselves, introduce fast ions into the plasma which also contribute to the ion orbit losses ${ }^{13,14}$. The fluxes of fast ion losses can be measured and allow for a direct connection to experimental efforts ${ }^{8,20}$.

Various ion orbit loss models have been designed to study the contribution of such losses to electric field generation $^{10,21}$, the distribution of ions entering the $\mathrm{SOL}^{22}$, and the intrinsic toroidal rotation associated with the preferred loss of counter-current ions ${ }^{23-27}$. Our aim is to continue this research in order to find a model for ion orbit loss in a realistic axi-symmetric magnetic field geometry while accounting for the effects of a pre-existing radial electric field, to characterize the ion losses while providing a physical description for how ion orbit loss contributes to the pedestal formation and the radial electric field.

To best capture the effects of ion orbit loss, our model makes use of experimentally determined equilibria and profiles. In this work, experimental data from two ASDEX Upgrade discharges are investigated: 30628 and 31533. As a generator of radial electric field, selfconsistency requires that the effects of any pre-existing radial electric field be carefully considered, highlighting the importance of precise experimental measurements of $E_{r}{ }^{28,29}$. Two instances in time are chosen for shot 30628 : one during the L-mode just before the L-H transition and the other in the resultant $\mathrm{H}$-mode. A single timestamp for ASDEX Upgrade discharge 31533, which has similar plasma parameters but a higher ion temperature profile, is also studied.

We first develop the ion orbit loss model in generality. Along the way, for clarity, illustrations are made using experimental shot data. We approach the problem beginning with the guiding center constants of motions, 
similarly to many of our predecessors ${ }^{11-15,21,23}$. After developing a set of equations and conditions that guide our application of said equations, we define two motivated and non-mutually exclusive definitions of ion orbit loss. Once the model has been established, the calculations are applied to the three experimental data sets mentioned above. A qualitative discussion concerning the future dynamic treatment of ion orbit loss concludes the work.

\section{The Constraint Surface}

Following previous investigations of ion orbit loss, we begin by considering the three constants of motion associated with the guiding center motion of an ion in an axi-symmetric magnetic field:

$$
\begin{aligned}
E & =\frac{1}{2} m v^{2}+q \Phi=\frac{1}{2} m v_{0}^{2}+q \Phi_{0} \\
\mu & =\frac{m v_{\perp}^{2}}{2 B}=\frac{m v_{\perp 0}^{2}}{2 B_{0}} \\
& =\frac{m v^{2}}{2 B}\left(1-\zeta^{2}\right)=\frac{m v_{0}^{2}}{2 B_{0}}\left(1-\zeta_{0}^{2}\right) \\
p_{\phi} & =m R v_{\|} f_{\phi}+q \psi=m R_{0} v_{\| 0} f_{\phi 0}+q \psi_{0} \\
& =m R \zeta v f_{\phi}+q \psi=m R_{0} \zeta_{0} v_{0} f_{\phi 0}+q \psi_{0},
\end{aligned}
$$

where $m$ and $q$ are the ion's mass and charge, $R$ is the distance from the center line of the tokamak, $B$ is the magnitude of the magnetic field, $\Phi$ is the electric potential, $\psi$ is the poloidal flux, $v$ is the ion's velocity, $v_{\phi}$ is the toroidal velocity, $v_{\|}=\vec{v} \cdot \vec{B} / B$ is the parallel velocity, $v_{\perp}$ is the perpendicular velocity, $\zeta=\frac{v_{\|}}{v}$ is the cosine of the pitch angle, and $f_{\phi}=\frac{B_{\phi}}{B}$. The spatial quantities are all evaluated at the guiding center position. Note, the final form of Eq. (3) enforces that $v_{0}$ is a positive quantity.

For a collisionless trajectory, the energy, $E$, is an exact invariant. The magnetic moment, $\mu$, is the well known adiabatic invariant. The toroidal canonical momentum, $p_{\phi}$, is an exact invariant in a perfectly axi-symmetric magnetic field. While the effects of toroidal symmetry breaking are an active area of research, axi-symmetry is assumed for simplicity.

The energy and canonical momentum are only perfectly conserved when evaluated at the particle position. The related guiding center constants of motion are conserved to a high degree; typically negligible differences, at least first order in the gyroradius, occur as a result of evaluation at the guiding center position rather than the instantaneous particle position ${ }^{30}$. Furthermore, the guiding center constants of motion typically omit the lowest order corrections for the perpendicular drifts. The first magnetic moment correction is first order in the gyroradius, and $\mu$ is reasonably conserved for thermal ions in a field configuration resembling that of a tokamak ${ }^{30,31}$. The first correction for the canonical momentum is second order ${ }^{30}$.
The constants of motion are often used to find the parallel velocity for an ion's guiding center,

$$
v_{\|}\left(R, z ; E, \mu, p_{\phi}\right)= \pm v_{0} \sqrt{R_{b}^{*}+\frac{2 q}{m v_{0}^{2}}\left(\Phi_{0}-\Phi\right)},
$$

where

$$
R_{b}^{*}=1-\frac{B}{B_{0}}\left(1-\zeta_{0}^{2}\right),
$$

as a function of its position in the poloidal plane, characterized by coordinates $(R, z)$. $R_{b}^{*}$ defines the toroidal bounce position in the case of negligible radial electric field.

We further rearrange the constants of motion to develop a geometric representation of the guiding center orbit. Two functional surfaces, labeled $\psi_{+}$and $\psi_{-}$, are found that inform which values of the poloidal flux an ion can experience such that its constants of motion remain unchanged. Together, these two functional surfaces form the constraint surface,

$$
\begin{aligned}
& \psi_{ \pm}\left(R, z ; R_{0}, z_{0}, v_{0}, \zeta_{0}\right)= \\
& \quad \frac{1}{q}\left(p_{\phi} \pm m R v_{0} f_{\phi} \sqrt{R_{b}^{*}+\frac{2 q}{m v_{0}^{2}}\left(\Phi_{0}-\Phi\right)}\right) .
\end{aligned}
$$

We use the lower-case $\psi$ for the constraint surface to emphasize that it is derived from Eqs. (1)-(3).

This surface contains different information than the equilibrium poloidal flux determined by the GradSchlüter-Shafranov equation,

$$
R^{2} \nabla \cdot\left(R^{-2} \nabla \Psi_{\mathrm{pol}}\right)=-I{\frac{d I}{d \Psi_{\mathrm{pol}}}}-R^{2} \mu_{0} \frac{d p}{d \Psi_{\mathrm{pol}}},
$$

where $I=R B_{\phi}$ and $p$ is the pressure. The above defines another surface in $(R-z-\psi)$-space which represents the flux prescribed by the MHD equilibrium. This physical flux further regulates the poloidal flux values that an ion will truly experience throughout an orbit. In other words, the intersection between $\Psi_{\text {pol }}$ and $\psi_{ \pm}$defines the allowed guiding center orbit.

The constraint surface allows for the determination of the initial energy needed for an ion to have an orbit which intersects the separatrix at some loss point $\mathcal{P},\left(R_{L}, z_{L}\right)$. Rearranging Eq. (5) provides the initial kinetic energy required for the intersection to take place,

$$
\begin{aligned}
E= & \frac{q^{2}}{2 m \xi^{2}}\left[\frac{R_{0}}{R^{2}} \frac{f_{\phi 0}}{f_{\phi}^{2}} \zeta_{0}\left(\psi_{\text {sep }}-\psi_{0}\right)\right. \\
& \left. \pm \sqrt{R_{b}^{*} \frac{\left(\psi_{\mathrm{sep}}-\psi_{0}\right)^{2}}{f_{\phi}^{2} R^{2}}+\frac{2 m}{q} \xi\left(\Phi_{0}-\Phi\right)}\right]_{\mathcal{P}}^{2},
\end{aligned}
$$

where

$$
\xi=\frac{R_{0}^{2}}{R^{2}} \frac{f_{\phi 0}^{2}}{f_{\phi}^{2}} \zeta_{0}^{2}-R_{b}^{*}
$$


(a)

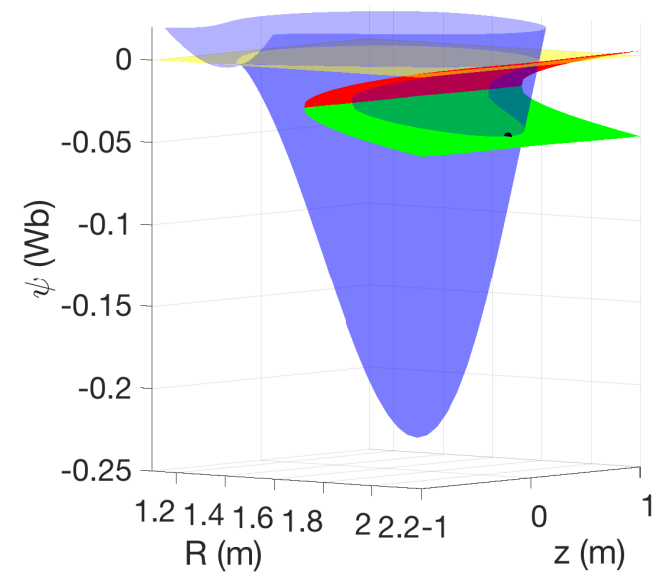

(b)

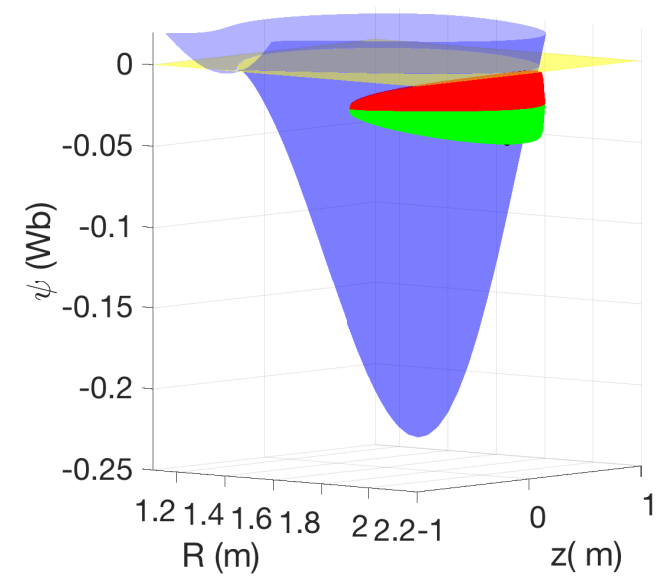

FIG. 1. A sample constraint surface is plotted in the case of radial electric field neglect (a) and inclusion (b). The initial position $\left(R_{0}, z_{0}, \psi_{0}\right)$ is indicated by the black dot, $\Psi_{\text {pol }}$ is in blue, and $\psi_{ \pm}$is in red and green. The equilibrium poloidal flux is that of ASDEX Upgrade shot 31533 at $t=3.0 \mathrm{~s}$.

The ambiguity found within the ' \pm ' sign is clarified by separately finding the minimum energy for the intersection to occur with either $\psi_{+}$,

$$
\begin{aligned}
E_{+}= & \frac{q^{2}}{2 m \xi^{2}}\left[\frac{R_{0}}{R^{2}} \frac{f_{\phi 0}}{f_{\phi}^{2}} \zeta_{0}\left(\psi_{\mathrm{sep}}-\psi_{0}\right)\right. \\
& \left.-\sqrt{R_{b}^{*} \frac{\left(\psi_{\mathrm{sep}}-\psi_{0}\right)^{2}}{f_{\phi}^{2} R^{2}}+\frac{2 m}{q} \xi\left(\Phi_{0}-\Phi\right)}\right]\left.\right|_{\mathcal{P}} ^{2},
\end{aligned}
$$

or with $\psi_{-}$,

$$
\begin{aligned}
E_{-}= & \frac{q^{2}}{2 m \xi^{2}}\left[\frac{R_{0}}{R^{2}} \frac{f_{\phi 0}}{f_{\phi}^{2}} \zeta_{0}\left(\psi_{\mathrm{sep}}-\psi_{0}\right)+\right. \\
& \left.+\sqrt{R_{b}^{*} \frac{\left(\psi_{\mathrm{sep}}-\psi_{0}\right)^{2}}{f_{\phi}^{2} R^{2}}+\frac{2 m}{q} \xi\left(\Phi_{0}-\Phi\right)}\right]\left.\right|_{\mathcal{P}} ^{2} .
\end{aligned}
$$

As noted before, our definition of $p_{\phi}$ assumes the positiveness of $v_{0}$. For the energy to be consistent with pre- vious definitions, the bracketed quantities in Eq. (8) and Eq. (9) must be positive as well. This is shown to be a sufficient condition in Appendix B.

As a brief aside, consider how an ion's initial conditions inform its initial position on $\psi_{ \pm}$. Evaluating $\psi_{ \pm}\left(R_{0}, z_{0}\right)$ indicates that

$$
\begin{aligned}
\psi_{ \pm}\left(R_{0}, z_{0}\right) & =\frac{1}{q}\left(p_{\phi} \pm m R_{0} v_{0} f_{\phi 0} \sqrt{\zeta_{0}^{2}}\right) \\
& =\frac{1}{q}\left(m R_{0} \zeta_{0} v_{0} f_{\phi 0}+q \psi_{0} \pm m R_{0} v_{0}\left|\zeta_{0}\right| f_{\phi 0}\right) \\
& =\psi_{0} .
\end{aligned}
$$

The previous is only true if the ' + ' corresponds to $\zeta_{0} \leq 0$ and the '-' to $\zeta_{0}>0$. The former begin on $\psi_{+}$and the latter on $\psi_{-}$. Without loss of generality, we have made the arbitrary choice to consider ions with $\zeta_{0}=0$ as initially being on $\psi_{+}$.

Eq. (8) and Eq. (9) do not provide freedom but rather the requirement of determining when each is valid for application. It is clearly seen in Fig. 1, that the minimum energy for the ion orbit to intersect the LCFS coincides with the first occurrence of an intersection of the upper surface and the separatrix. In the case of ions with initial conditions placing them on the lower surface, whether a transfer from the lower surface to the upper can take place should be taken into consideration. To ensure the correct threshold loss energies are found, orbit type accessibility for differing ions must be classified.

\section{Banana Orbit Accessibility}

Throughout this discussion, we will refer to the specific case presented in Fig. 1: $\psi_{\text {sep }}>\psi_{0}$ and $\psi_{+} \geq \psi_{-}$. In generality, the discussion can be convoluted as the arbitrary orientations of $B_{\phi}$ and $I_{p}$ introduce subtle sign changes. For example, $B_{\phi}<0$ implies that $\psi_{-} \geq \psi_{+}$. Furthermore, the signs of $B_{\phi}$ and $I_{p}$ influence whether $\psi_{\text {sep }}$ is lesser or greater than the interior, in order for the definition of $p_{\phi}$ to be consistent.

In this configuration, an ion beginning its orbit on $\psi_{+}$, $\zeta_{0} \leq 0$, will reach the LCFS with minimum energy at the first intersection of $\psi_{+}$with the separatrix, since $\psi_{+}>$ $\psi_{-}$. If an ion begins its orbit on $\psi_{-}, \zeta_{0}>0$, the threshold loss energy will occur under the same condition as long as $\Psi_{\text {pol }}$ allows for a banana orbit to take place, otherwise the threshold will occur at the first intersection of $\psi_{-}$ with the separatrix.

A transfer from $\psi_{-}$to $\psi_{+}$must occur when $\psi_{-}=\psi_{+}=$ $p_{\phi} / q$. To determine if an ion with $\zeta_{0}>0$ will exhibit a banana orbit, we find the contour of $\Psi_{\text {pol }}$ where a transfer would occur, $\Psi_{\text {pol }}=p_{\phi} / q$. The rooted quantity in Eq. (5) is used to determine if there is a point where the constraint surface intersects $\Psi_{\mathrm{pol}}$. Making use of the fact 
that in a tokamak $B(R, z) \approx B(R) \sim 1 / R$, one sees that

$$
\begin{aligned}
& 1-\frac{B\left(R_{b}, z_{b}\right)}{B_{0}}\left(1-\zeta_{0}^{2}\right)+\frac{2 q}{m v_{0}^{2}}\left(\Phi_{0}-\Phi\left(\rho_{b}, \theta_{b}\right)\right)=0 \\
& 1-\frac{R_{0}}{R_{b}}\left(1-\zeta_{0}^{2}\right)+\frac{2 q}{m v_{0}^{2}}\left(\Phi_{0}-\Phi\left(\rho_{b}, \theta_{b}\right)\right) \approx 0 \\
& R_{b}\left(\rho_{b}, \theta_{b}\right) \approx \frac{R_{0}\left(1-\zeta_{0}^{2}\right)}{1+\frac{2 q}{m v_{0}^{2}}\left(\Phi_{0}-\Phi\left(\rho_{b}, \theta_{b}\right)\right)}
\end{aligned}
$$

where the subscript ' $b$ ' is used to represent variables evaluated at the toroidal bounce point, $\theta$ is the poloidal angle measured from the outer-midplane, and $\rho$ is a dimensionless radial coordinate which labels flux surfaces.

For simplicity, the profiles of the electric potential and temperature are taken to be only dependent on the dimensionless radial coordinate, $\rho_{\text {pol }}$, defined in Eq. (18). The toroidal bounce location can be further specified as it must occur at the $\rho$ corresponding to the contour $\Psi_{\mathrm{pol}}=p_{\phi} / q$

$$
R_{b} \approx \frac{R_{0}\left(1-\zeta_{0}^{2}\right)}{1+\frac{2 q}{m v_{0}^{2}}\left(\Phi_{0}-\Phi\left(\rho\left(\Psi_{\mathrm{pol}}=p_{\phi} / q\right)\right)\right)} .
$$

The bounce location is dependent on both the geometry of the magnetic and electric fields and the initial conditions of the ion and will be used to find the accessibility of banana orbits for counter-current ions initiated with some $\left(R_{0}, z_{0}, \zeta_{0}\right)$, or alternately $\left(\rho_{0}, \theta_{0}, \zeta_{0}\right)$.

The relevant energy range for banana losses is lower bounded by the energy needed to escape the electric potential,

$$
E_{B_{\min }}=q\left(\Phi_{\text {sep }}-\Phi_{0}\right),
$$

and is upper bounded by the energy for the bounce to occur at the same poloidal flux value as the LCFS,

$$
E_{B_{\max }}=\frac{1}{2} m q^{2}\left(\frac{\psi_{\mathrm{sep}}-\psi_{0}}{m R_{0} \zeta_{0} f_{\phi 0}}\right)^{2} .
$$

Although a more energetic, yet similar, ion may technically travel on a banana orbit, the ion would cross the separatrix before the toroidal bounce and is captured by Eq. (9).

For a banana orbit to occur, Eq. (11) must return a value for $R_{b}$ that is valid to intersect the contour $\Psi_{\mathrm{pol}}=$ $p_{\phi} / q ; R_{b}$ needs to be larger than the minimum position on the contour,

$$
\mathcal{R}_{1} \equiv R_{b}(E)-R_{\min }\left(\Psi_{\text {pol }}=p_{\phi}(E) / q\right) \geq 0,
$$

and smaller than the maximum,

$$
\mathcal{R}_{2} \equiv R_{\max }\left(\Psi_{\text {pol }}=p_{\phi}(E) / q\right)-R_{b}(E) \geq 0 .
$$

For electric potential profiles typical in the edge of a tokamak, like those seen in Fig. 5, there are four possible options for an ion with initial conditions $\left(\rho_{0}, \theta_{0}, \zeta_{0}\right)$. (a)

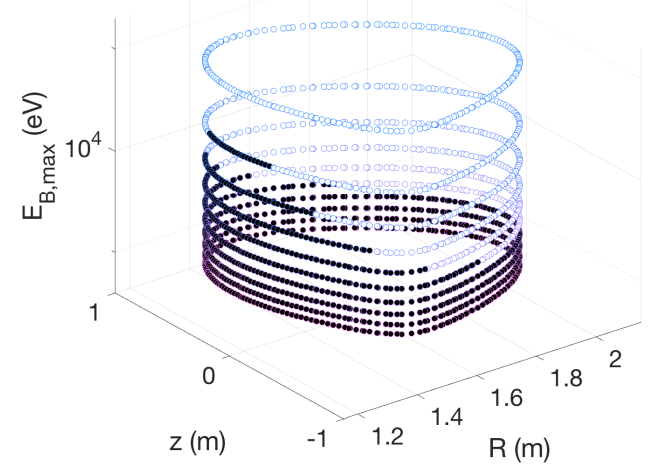

(b)

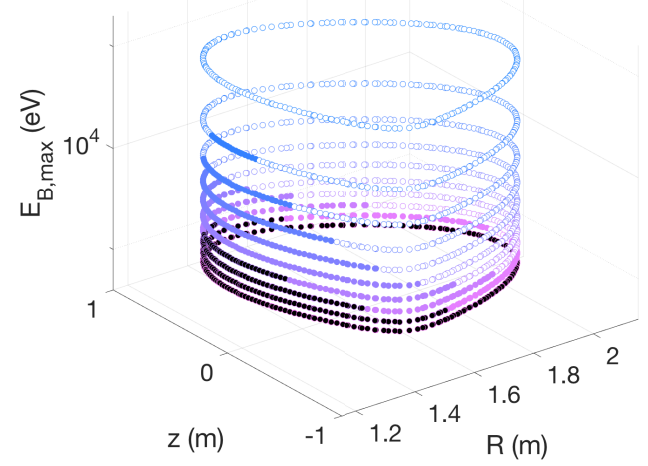

FIG. 2. The results of following the procedure outlined in Section III. for ASDEX Upgrade shot 31533 at $t=3.0 \mathrm{~s}$ for the $\rho_{0}=0.98$ flux surface in the cases of radial electric field neglect (a) and inclusion (b). White-faced color-edged circles correspond to option 3, full banana orbit accessibility. Color-faced color-edged circles corresponds to option 4, finite energy banana orbit accessibility. Black-faced color-edged circles correspond to 2, no banana orbit accessibility. For colors, see Fig. 6.

1. If $E_{B_{\min }}>E_{B_{\max }}$, an ion cannot be lost via $\psi_{+}$due to the electric potential. We must find a loss energy using Eq. (9) that is able to escape the potential well.

2. If $\mathcal{R}_{1}<0$ or $\mathcal{R}_{2}<0 \forall E_{B_{\min }} \leq E \leq E_{B_{\max }}$, then any ion within the relevant range cannot be lost via a banana orbit. We must find the minimum energy using Eq. (9).

3. If $\mathcal{R}_{1} \geq 0$ and $\mathcal{R}_{2} \geq 0 \forall E_{B_{\min }} \leq E \leq E_{B_{\max }}$, then any ion within the relevant range of energies has the potential for loss via a banana orbit. Or if $\mathcal{R}_{1} \geq 0 \forall$ $E_{B_{\min }} \leq E \leq E_{B_{\max }}$ and $\mathcal{R}_{2} \geq 0 \forall E_{B_{\min } \tilde{E}_{R}}<\tilde{E}_{R_{\mathrm{lb}}} \leq$ $E \leq E_{B_{\max }}$, then any ion within the range $\tilde{E}_{R_{\mathrm{lb}}} \leq E \leq$ $E_{B_{\max }}$ can be lost via a banana orbit, corresponding to energies given by Eq. (8).

4. Else, we find that there is a finite range of energies that 
allow for banana orbits. The electric field allows for a fraction of ions in the relevant range to banana orbit while more energetic ions exhibit passing orbits.

It should be noted that the above procedure is approximate. For any set of initial conditions, one could in principle find the exact guiding center orbit. Doing so for all relevant ions would be unavoidably more expensive. Due to the geometries of $\Psi_{\text {pol }}$ and $\psi_{ \pm}$, nearly fieldperpendicular counter-current ions starting from very near the midplane will have a slight over-estimation in their trapped orbit accessibility. The results should not qualitatively or quantitatively be appreciably altered.

Fig. 2 shows the effects of the radial electric field on orbit type accessibility. Greater areas of configurationspace have full access to banana orbits for more initial pitch directions and now many regions have energetically bounded access to such orbits. A stronger radial field has a more pronounced effect and for large enough magnitudes can inhibit banana orbit accessibility, firstly for ions starting near the outer-midplane.

In the following subsection, option 4 . is discussed in more detail. Unlike the other cases, for $\left(\rho_{0}, \theta_{0}, \zeta_{0}\right)$ falling into case 4., the choice of application of Eq. (8) or Eq. (9) is in itself energy dependent.

\section{A. Banana Loss Islands (BLIs)}

For some $\left(\rho_{0}, \theta_{0}, \zeta_{0}\right)$, the radial electric field allows for a finite range of energies which correspond to banana orbits; energies above some upper bound are passing orbits. The required energy for a counter-current ion to cross the LCFS is significantly lowered if the ion will travel on a banana orbit. Therefore any tokamak plasma that has a radial electric field in its edge, generically corresponding to a positive potential increase with $\rho$, will have isolated regions in velocity-space that correspond to toroidally trapped orbits. If the trapped orbits are lost out of the plasma, the associated loss holes bleed out relatively low energy counter-current ions. These loss islands sit underneath populated regions of velocity-space corresponding to higher energy ions and are likely to refill under thermal relaxation.

Fig. 3 visualizes orbits representative of this phenomenon. Here, the equilibrium and profiles for ASDEX upgrade shot 31533 , which are detailed in section V., are used. The red and green lines correspond to the intersection of $\psi_{+}$and $\psi_{-}$with $\Psi_{\text {pol }}$ in the same way as in Fig. 1. The radial electric field has a pronounced effect on the three-dimensional behavior of the orbits. In a typical banana orbit where the effects of $E_{r}$ are unimportant, the poloidal and toroidal bounce positions are taken to be the same. It is known that near the separatrix, the strong radial electric field can reverse the toroidal rotation direction ${ }^{32}$. The top plot illustrates a similar effect happening to a single ion. The radial electric field decouples the orbit's poloidal and toroidal bounce locations, allowing for the pictured banana orbit. A more (a)

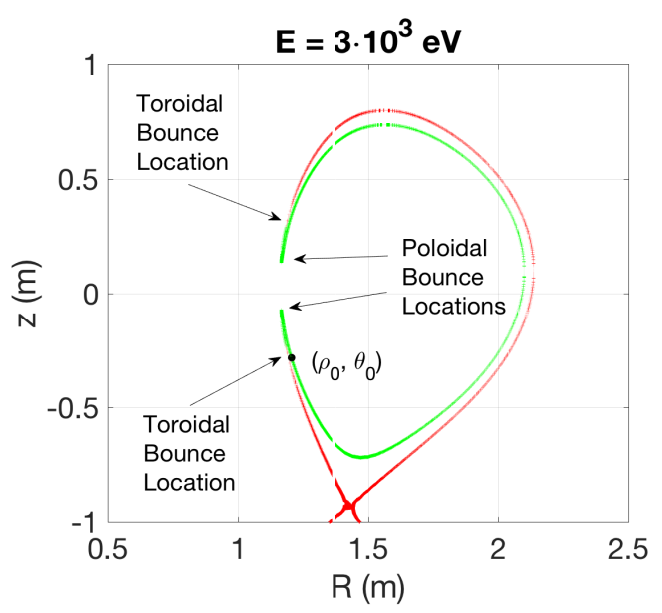

(b)

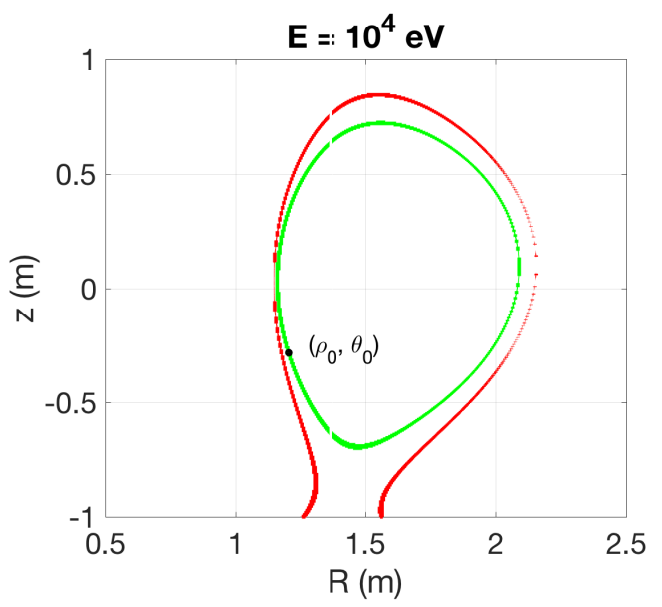

FIG. 3. Two ion guiding center orbits starting from the same poloidal angle on the $\rho_{0}=0.98$ flux surface, one existing in a BLI (a) and the other just above (b).

energetic ion, with the same $\left(\rho_{0}, \theta_{0}, \zeta_{0}\right)$, has a receded toroidal bounce position which is no longer accessible, see Eq. (11), and the counter-current ion cannot transfer from $\psi_{-}$to $\psi_{+}$. The ion is forced onto a passing orbit.

Although the instance in Fig. 3 does, there is nothing that guarantees that an ion with an electric-fieldallowed banana orbit will be lost. We must always check if any of the energies within this range correspond to orbits that fit a definition of lost. If they do, then there is an upwardly bounded region of velocity-space from which counter-current ions leave the plasma. We refer to these as banana loss islands (BLIs) to highlight their disconnectedness from the upwardly unbounded velocity-space loss holes associated with the threshold loss energies.

For various initial $\left(\rho_{0}, \theta_{0}, \zeta_{0}\right)$ we have developed a method to determine if the lowest energy required for energetically unbounded ion loss, the threshold ion loss energy, is given by Eq. (8) or Eq. (9). There are also bounded loss regions in velocity-space, BLIs, which correspond to an energy dependence in appropriate application of either Eq. (8) or Eq. (9). Before characterizing the velocity-space loss hole for the plasma, it is important to establish what is meant by 'loss'. 


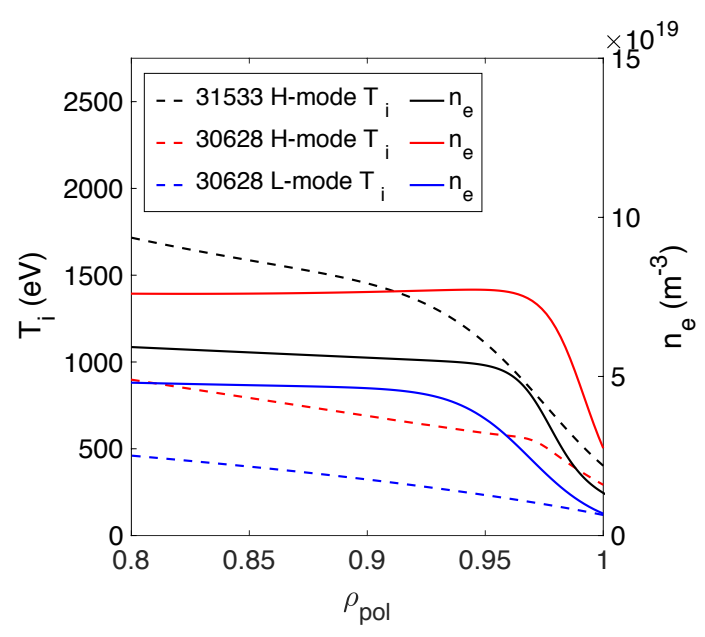

FIG. 4. Experimental electron density and ion temperature profiles for ASDEX Upgrade discharges.

\section{Lost Orbit Definitions}

Thus far, we have simply found expressions for the required energy an ion must possess in order to have its guiding center orbit intersect some position on the LCFS. Two definitions for ion orbit loss will be proposed: one is inclusive while the other is more strict.

The first considers all orbits which intersect the separatrix at any point to be lost. These so called 'lost' ions will sample the scrape off layer (SOL). Some fraction will either backscatter into the plasma or will re-enter the LCFS on an unperturbed orbit ${ }^{21}$.

The second definition only considers ions which are lost via the $\mathrm{X}$-point. Ion $\mathrm{X}$-loss is kinetically associated with a poloidal trapping of the orbit in the region of low poloidal $B$ near the X-point ${ }^{16}$. The poloidally trapped ions suffer a prolonged exposure to the unchecked effects of the $\nabla B$ and curvature drifts. Insofar as a guiding center theory is correct, these orbits are captured by the geometries of $\psi_{ \pm}$and $\Psi_{\text {pol }}$. X-lost ions spend little time in the SOL and are not expected to re-enter the plasma. Since ions lost very near the $\mathrm{X}$-point behave similarly ${ }^{17}$, ions intersecting the separatrix within $0.1 \mathrm{rad}$ of the $\mathrm{X}$ point are taken to be X-lost. All X-lost orbits are also captured by the SOL sampling definition for ion orbit loss.

It is known that the forward $\nabla B$ configuration, ion drifts pointing towards the X-point, results in a more pronounced $E_{r}$ generation ${ }^{5,17}$. In the case of backward $\nabla B$, the X-lost ions cross the LCFS at the opposite side of the plasma and are maximally exposed to the SOL effects mentioned above. Radial electric field generation is not null in the unfavorable drift configuration, implying that some fraction of the SOL sampling ions that cross the LCFS away from the X-point must truly be lost. To that end, it is important to consider both loss classes when characterizing ion orbit loss. (a)

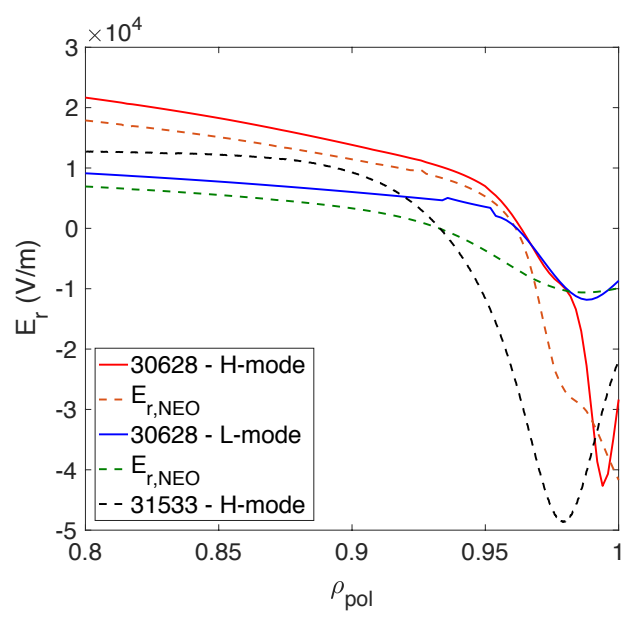

(b)

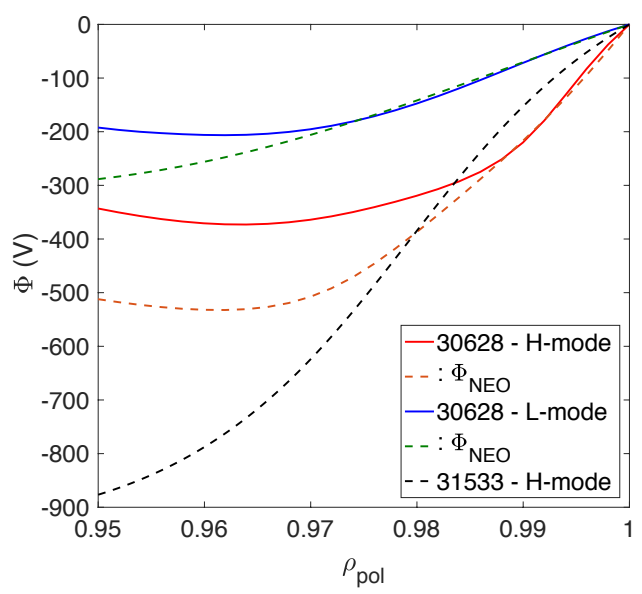

FIG. 5. Experimental (solid) and calculated (dashed) radial electric field (a) and electric potential (b) profiles for ASDEX Upgrade discharges.

\section{Ion Orbit Loss}

The aforementioned machinery is used to investigate ion orbit loss for both SOL sampling ions and for X-lost ions. Recall, Eq. (8) and Eq. (9) respectively provide the minimum energies such that $\psi_{+}$and $\psi_{-}$intersect with some given point on the separatrix. A unique ion is categorized by its initial conditions, $\left(\rho_{0}, \theta_{0} ; E, \zeta_{0}\right)$.

Therefore, ions with the same $\left(\rho_{0}, \theta_{0}, \zeta_{0}\right)$ are only differentiated by their energy; for some energies the ions are confined whereas for others the ions are lost. As detailed in Section III., for each unique $\left(\rho_{0}, \theta_{0}, \zeta_{0}\right)$ we need to ensure which type of orbit can take place.

For the SOL sampling ions, we scan over a series of loss points along the separatrix and take the minimum intersection energy to be the threshold loss energy,

$$
E_{T}=\min \left(E_{+} \delta_{+}+\left.E_{-} \delta_{-}\right|_{\mathcal{P}} \forall \mathcal{P} \in \mathbf{L C F S}\right)
$$

In the above, $\delta_{+}$and $\delta_{-}$are respectively zero if the threshold energy corresponds to either a loss on $\psi_{-}$or $\psi_{+}$, according to the procedure outlined in Section III. 
The X-loss thresholds are found by simply finding the minimum energy for an ion to be lost very near the Xpoint, see Section IV. In both cases, the BLIs are not included in the calculations; the definition of a threshold loss energy ensures that any more energetic, yet otherwise identical, ion will also be lost. For the $\left(\rho_{0}, \theta_{0}, \zeta_{0}\right)$ determined to support banana orbits for a finite range of energies, the BLI associated energy range is separately checked for losses.

Experimentally determined equilibria data and radial profiles from two ASDEX Upgrade discharges, 30628 $\left(B_{\phi}=-2.494 T, I_{p}=1.002 M A, \bar{R}=1.68 \mathrm{~m}\right)$ and 31533 $\left(B_{\phi}=-2.465 T, I_{p}=1.004 M A, \bar{R}=1.71 \mathrm{~m}\right)$, are investigated. We study two instances in time for shot 30628: $t=1.6 \mathrm{~s}$ corresponds to L-mode conditions just before the $\mathrm{L}-\mathrm{H}$ transition and $t=3.5 \mathrm{~s}$ to those of the $\mathrm{H}$-mode. Also considered is the H-mode discharge 31533, taken at $t=3.0 \mathrm{~s}$; discharge 31533 has an ion temperature profile considerably higher than that of shot 30628 allowing for a look into ion temperature sensitivity. Since $B_{\phi}$ and $I_{p}$ are oppositely signed in both discharges, $\zeta_{0}>0$ corresponds to counter-current ions while $\zeta_{0}<0$ to co-current. The orientations of the toroidal field and poloidal current also inform that $\psi_{-} \geq \psi_{+}$and $\psi_{0}>\psi_{\text {sep }}$, ensuring consistency with previous definitions.

For comparison, the neoclassically predicted radial electric fields are also considered ${ }^{33}$,

$$
E_{\mathrm{NEO}}=\frac{T_{i}}{q}\left(\frac{1}{h_{\rho}} \frac{1}{n_{i}} \frac{d n}{d \rho}+\frac{k_{t}}{h_{\rho}} \frac{1}{T_{i}} \frac{d T_{i}}{d \rho}\right)
$$

where $T_{i}$ is the ion temperature, $n_{i}$ is the ion number density, $k_{t} \sim 1$ reflects the plasma's regime of collisionality, and $h_{\rho}$ is the scale factor necessary in the dimensionless coordinates. In the above, the terms relating to the main ion rotation are neglected. The poloidal rotation is small in the neoclassical approximation, and the toroidal rotation contributes little in the edge; both contribute negligibly to the edge radial electric field when compared to the main ion gradients ${ }^{34}$. Studying the neoclassical electric fields allows us to investigate the effects of subtle electric potential profile changes.

The threshold ion loss energies and BLIs for deuterium ions initiated at different poloidal locations along various initial flux surfaces, labeled using the poloidal flux, are found. The dimensionless radial coordinate,

$$
\rho_{\mathrm{pol}} \equiv \sqrt{\frac{\Psi_{\mathrm{pol}}-\Psi_{\mathrm{axis}}}{\Psi_{\mathrm{sep}}-\Psi_{\mathrm{axis}}}},
$$

is defined such that $\rho=0$ lies on the magnetic axis and $\rho=1$ corresponds to the LCFS. Since the threshold energies are found for various $\left(\rho_{0}, \theta_{0}, \zeta_{0}\right)$, we choose to represent the initial pitch directions via color and typically in the plots hold either $\rho_{0}$ or $\theta_{0}$ constant while varying the other. Fig. 5 illustrates the chosen color gradient, as well as establishing the color representative for the ion temperature.

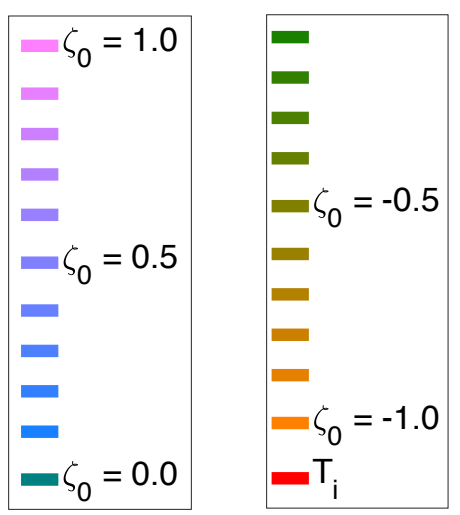

FIG. 6. Legend for Fig. 2 and Figs. 7-11. The color gradient spans over initial pitch directions, and the dashed red line represents the local ion temperature.

\section{A. Velocity-Space Loss Hole}

During the initial characterization of ion orbit loss, only the experimental data from the L-mode and H-mode of shot 30628 will be compared. Both $\mathrm{H}$-mode sets of data produce results which share many qualitative features. Discharge 31533 is included when the differences become illuminating.

\section{SOL Sampling Ions}

The threshold energies are found for ions to cross the LCFS for ASDEX Upgrade Shot 30628 in the L-mode and $\mathrm{H}$-mode. For each initial flux surface, $\rho_{0}$, the procedure outlined in Section III. is followed to determine for which $\left(\theta_{0}, \zeta_{0}\right)$ to apply either Eq. (8) or Eq. (9). Each instance is applied to different loss locations and the minimum of the energies is taken to be the threshold energy.

Fig. 7 shows the threshold energies for SOL sampling ions initiated from the $\rho_{0}=0.99$ flux surface for the two experimental data sets. Any ion located above the threshold energy line is considered lost. The plots are largely similar but contain telling differences.

Generically, counter-current ions are more likely to be lost when starting from near the outer-midplane, $\theta_{0}=0.0$, while co-current ions are typically only lost when starting from near the inner-midplane, $\theta_{0}=\pi$. The sudden jumps in the threshold ion energy correspond to the accessibility of banana losses. If able to toroidally bounce at all, counter-current ions with initial pitch closer to unity will only banana orbit when starting from nearer the outer-midplane. These results are in line with previous works ${ }^{22,23,26}$

The effects of the radial electric field are various and are most easily noted when comparing the $\mathrm{H}$ - and L-mode cases. There is an overall shift in the loss energies associated with the more sharply radially-increasing potential 
(a)

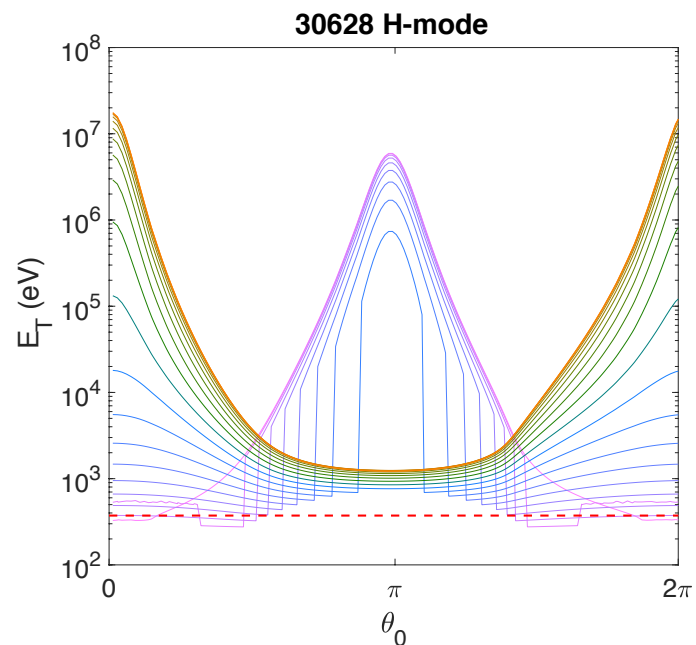

(b)

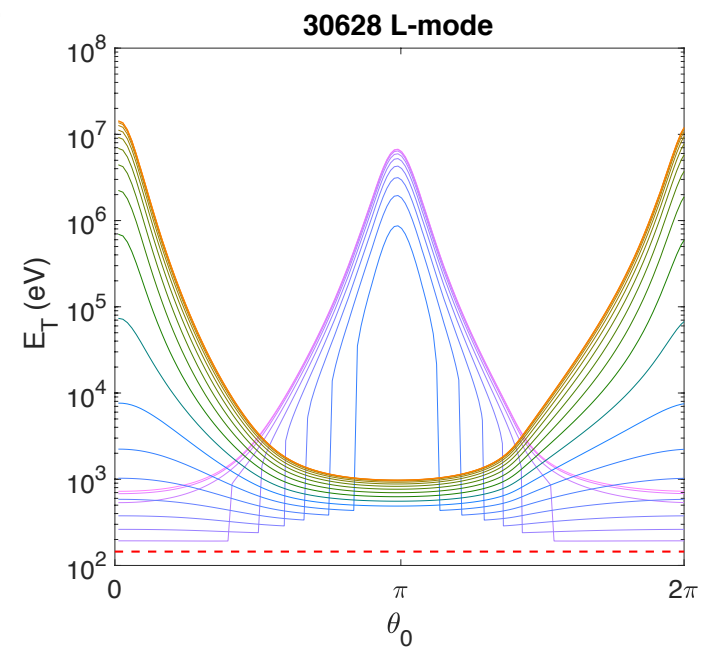

FIG. 7. The SOL sampling orbit threshold energies for ions initiated from flux surface $\rho_{0}=0.99$. See Fig. 6 for legend.

found in the edge of the $\mathrm{H}$-mode. The rise in the energies competes with the wider range of configuration-space in which counter-current ions are able to be lost on banana orbits in the presence of substantial electric fields typical in the edge, see Section III. A. In the case of the H-mode, counter-current ions starting from the $\rho_{0}=0.99$ flux surface with initial pitch direction as great as $\zeta_{0}=0.9$ have threshold loss energies corresponding to trapped orbits. In the L-mode, only counter-current ions up to $\zeta_{0}=0.7$ behave similarly. Another effect of $E_{r}$ is seen in the Hmode this near the edge of the plasma. The electric field inhibits the typically preferred banana losses for ions beginning at the outer-midplane, increasing the minimum loss energies. The effect is more easily seen in Fig. 9. A further difference between the two modes of operation is related to the increase of the local ion temperature in the $\mathrm{H}$-mode compared to the L-mode. Larger regions of the loss hole are thermally accessible to the H-mode plasma.

The BLIs first mentioned in Section III. A. are accounted for in Fig. 8. For the chosen discharge, these types of losses are seen close to the edge and informed (a)

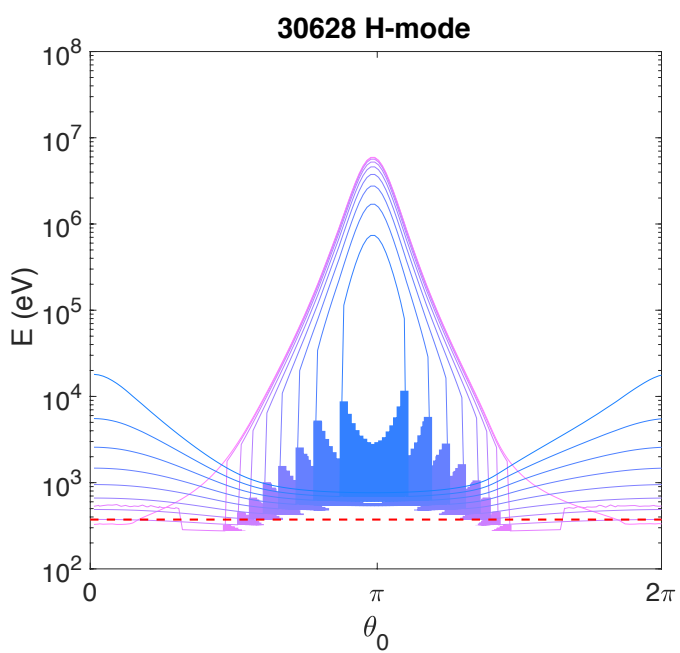

(b)

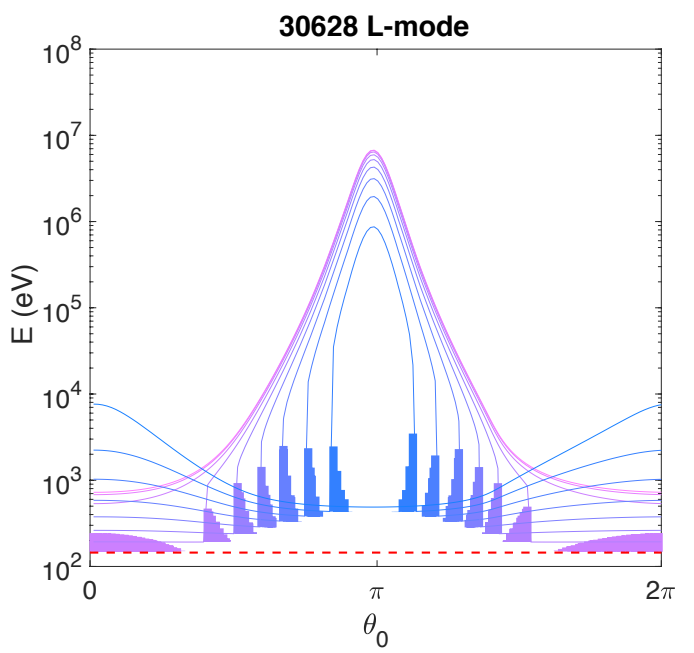

FIG. 8. The BLIs for SOL sampling loss orbits starting from the $\rho_{0}=0.99$ flux surface. The loss holes are plotted against the counter-current ion loss thresholds from Fig. 7. See Fig. 6 for legend.

the previous choice to look at the $\rho_{0}=0.99$ flux surface. For that reason, Fig. 8 plots the BLIs under the H- and L-mode conditions for shot 30628 for ions starting from the $\rho_{0}=0.99$ flux surface. For comparison, the loss islands are plotted against the threshold energies seen in Fig. 7. Any ion with initial pitch of matching color will be lost if found within a shaded region. In the case of the $\mathrm{H}$-mode, the loss islands are more significant and are concentrated nearer the inner-midplane. One can imagine that as the radial electric field increases in the edge, ions initiated nearer and nearer the inner-midplane are able to be toroidally trapped, as mentioned in Section III. While the field is developing, there would be an interim where lower energy ions are lost while more energetic ions are unable to exhibit banana orbits. In Eq. (11), for a given $\left(\rho_{0}, \theta_{0}, \zeta_{0}\right)$, there is only a single free parameter, that being the ratio of the potential change to the energy. A growing electric field corresponds to a growing potential difference. As the electric field grows in magnitude, 
(a)

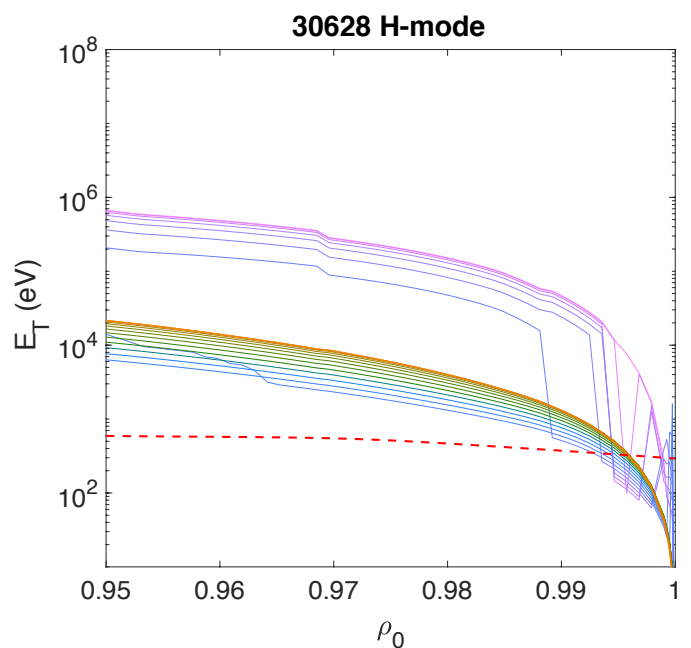

(b)

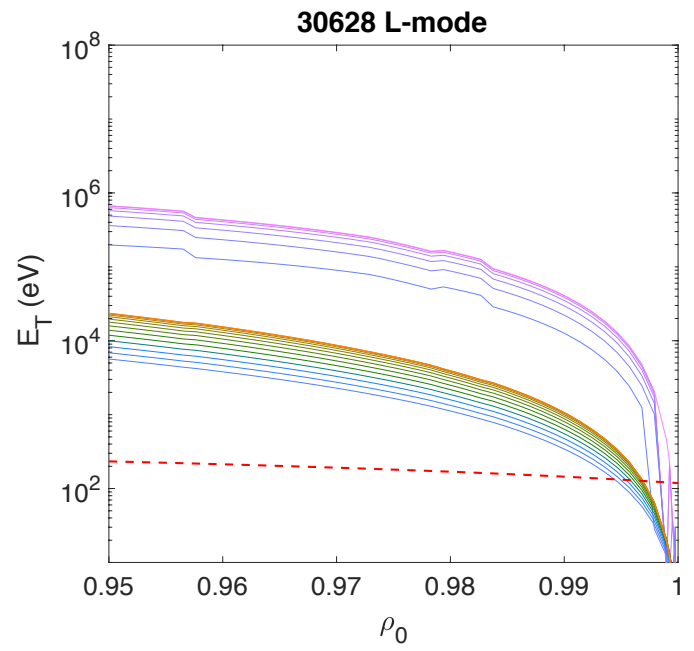

FIG. 9. The SOL sampling orbit threshold energies as a function of the radial coordinate for ions initiated from the outermidplane $\left(\theta_{0}=0\right)$. See Fig. 6 for legend.

the only way to leave Eq. (11) unchanged for a given $\left(\rho_{0}, \theta_{0}, \zeta_{0}\right)$ is to consider ions of increasing energy. Therefore, the upper bounds for the BLIs increase until they would theoretically open up to the rest of the velocityspace hole, provided there was a sufficiently growing $E_{r}$.

Fig. 9 illustrates the radial coordinate dependency for the SOL sampling threshold energies for ions found at the outer-midplane. Typically, ions nearer the separatrix have lower threshold loss energies. There is a clear preferential loss of counter-current ions for most of the flux surfaces with significant losses. As hinted at by Fig. 7 , nearer the edge counter-current ions are unable be lost on banana orbits, and as expected, the velocity-space loss hole for ions initiated at the outer-midplane is raised to higher energies in the presence of a strong electric field ${ }^{16}$. Due to the impact of said field on the ion orbits, the resultant threshold energy is often larger than that of a passing orbit loss in the case of negligible $E_{r}$. The preferential loss of counter-current ions is tempered by the radial electric field close to the LCFS; the peak of the (a)

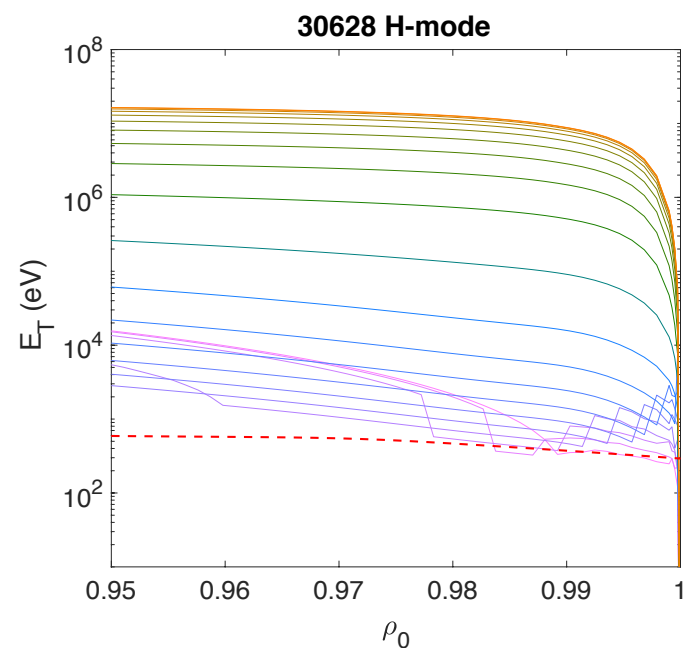

(b)

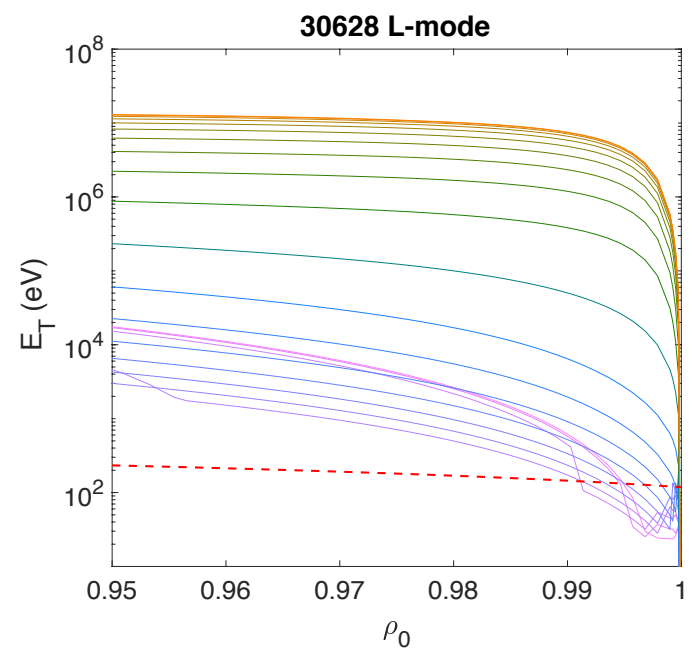

FIG. 10. The SOL sampling orbit threshold energies as a function of the radial coordinate for ions initiated from near the inner-midplane $\left(\theta_{0}=3 \pi / 4\right)$. See Fig. 6 for legend.

intrinsic toroidal rotation associated with ion orbit loss lies within the separatrix, as similarly expected ${ }^{27,35}$.

Fig. 10 is a companion to Fig. 9, only representing ions initiated near the inner-midplane $\left(\theta_{0}=3 \pi / 4\right)$. Here, $E_{r}$ often has the opposite effect; the radial electric field allows for trapped orbits and thereby lowers the threshold loss energies for local ions. The L- and H-mode losses are otherwise quite similar for co-current ions starting near the inner-midplane. The higher temperature profile in the H-mode still allow for greater thermal access to the loss hole than in the L-mode.

Now that we have found the velocity-space loss hole for ions that sample the SOL in any capacity, we narrow our view to ions that are lost near the $\mathrm{X}$-point. The discussion will be briefer to avoid redundancy since similar calculations are performed on a restricted set of loss locations. 


\section{X-lost Ions}

The velocity-space loss hole is now classified in the case of X-loss for both chosen instances of shot 30628. Again, this definition was selected for a more conservative view of ion orbit loss based on the fact that X-lost ions are unlikely to re-enter the plasma.

Fig. 11 plots both the threshold loss energies and the BLIs for X-lost ions starting from the $\rho_{0}=0.99$ flux surface. Similar to the case of SOL sampling orbits, the behavior of the co-current ions only sightly differs between L- and H-mode conditions. There is still the shift in the energies associated with the larger magnitude negative electric field in the edge of the H-mode. Now, the possibility of extremely energetic losses for co-current ions originating from the outer-midplane are disallowed because such passing orbits are never lost near the X-point, see Fig. 1. The behavior of the counter-current losses is subtler. For similar reasons mentioned above, the passing counter-current ions are more easily lost when initiated near the outer-midplane. For trapped ions, X-loss can only occur if the poloidal bounce point allows for loss near the X-point. For each initial pitch direction, only a selection of initial poloidal angles fit the criterion. Since the banana orbits associated with the BLIs typically toroidally bounce on the high field side, they are easily lost via the $\mathrm{X}$-point and are an important factor for X-loss.

Having found the loss regions in velocity-space for ion orbit loss in a few experimental configurations, we now calculate the fraction of lost ions, using both definitions. The fractions illustrate the significance of the thermal ion orbit losses in the pedestal.

\section{B. Loss Fractions}

By integrating the particle distribution function over the velocity-space loss hole, one determines the change in the ion density associated with ion orbit $\operatorname{loss}^{21}$,

$$
\begin{gathered}
n_{\mathrm{LOSS}}\left(\rho_{0}, \theta_{0}\right)=2 \pi \int_{-1}^{1} d \zeta_{0}\left[\int_{v_{T}\left(\rho_{0}, \theta_{0}, \zeta_{0}\right)}^{\infty} v_{0}^{2} f\left(\rho_{0}, v_{0}\right) d v_{0}+\right. \\
\left.\int_{v_{B_{L}}\left(\rho_{0}, \theta_{0}, \zeta_{0}\right)}^{v_{B_{H}}\left(\rho_{0}, \theta_{0}, \zeta_{0}\right)} v_{0}^{2} f\left(\rho_{0}, v_{0}\right) d v_{0}\right]
\end{gathered}
$$

from the original number density of ions,

$$
n\left(\rho_{0}\right)=4 \pi \int_{0}^{\infty} v_{0}^{2} f\left(\rho_{0}, v_{0}\right) d v_{0},
$$

where $f\left(\rho_{0}, v_{0}\right)$ is the particle distribution function. The lower bound of the first integral, $v_{T}$, corresponds to the velocity associated with the threshold loss energy while the bounds for the second integral, $v_{B_{L}}$ and $v_{B_{H}}$, correspond to the bounds of the BLIs, not to Eq. (12) and (a)

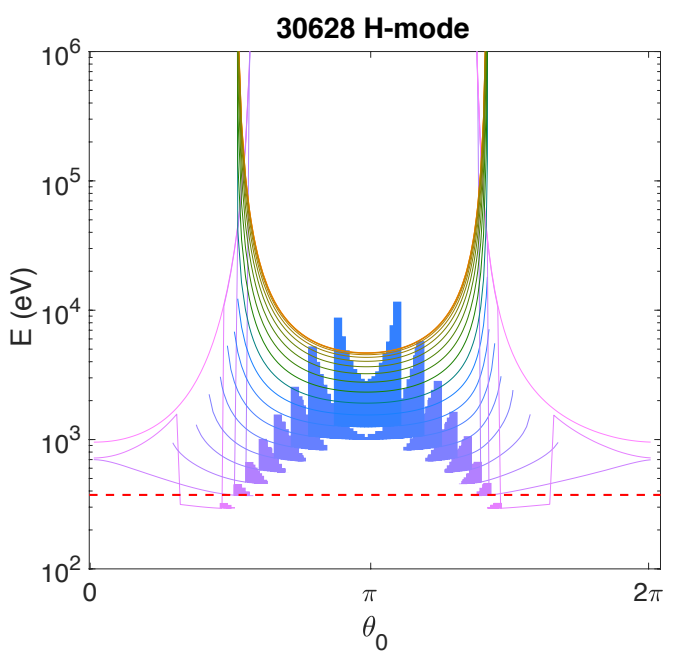

(b)

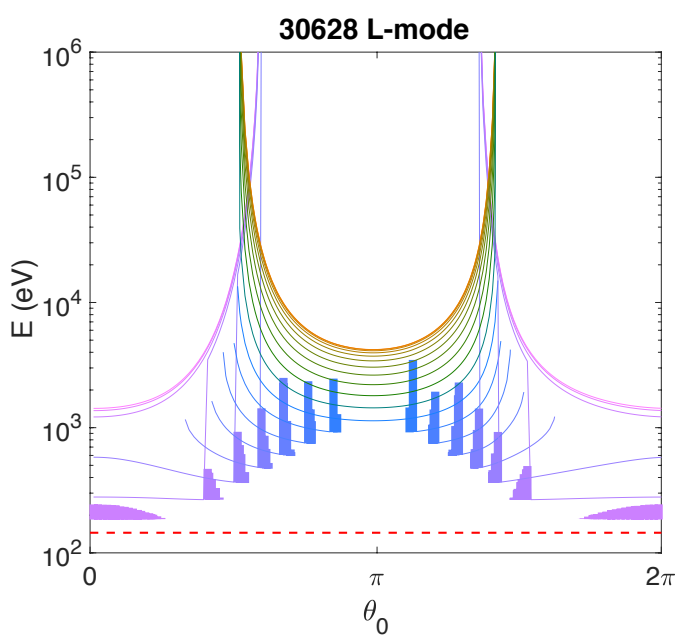

FIG. 11. The X-loss orbit threshold energies for ions initiated from flux surface $\rho_{0}=0.99$ plotted alongside the BLIs. For readability, most nearly vertical lines were omitted from the plot. See Fig. 6 for legend.

Eq. (13). We have assumed that the particle distribution function is initially isometric in velocity space; an ion is equally likely to have any initial pitch direction.

The poloidal-angle-dependent loss ratio for a given flux surface is the ratio of Eq. (19) and Eq. (20). Averaging over the flux surface determines the fraction of lost ions for a given $\rho_{0}$,

$$
\begin{aligned}
F\left(\rho_{0}\right) & =\frac{\int_{0}^{2 \pi} \int_{0}^{2 \pi} \frac{n_{\mathrm{LOSS}}}{n} \sqrt{g} d \phi d \theta}{\int_{0}^{2 \pi} \int_{0}^{2 \pi} \sqrt{g} d \phi d \theta} \\
& =\frac{\oint \frac{n_{\mathrm{LOSS}}}{n} \frac{d l}{B}}{\oint \frac{d l}{B}}
\end{aligned}
$$

where $\sqrt{g}$ is the Jacobian and the path integral is over a cross-section of the flux surface.

To continue, a distribution function, $f\left(\rho_{0}, v_{0}\right)$, must be specified. For simplicity, a simple Maxwellian distribu- 
tion,

$$
f\left(\rho_{0}, v_{0}\right)=n\left(\rho_{0}\right)\left(\frac{m}{2 \pi T_{i}}\right)^{3 / 2} e^{-m v_{0}^{2} / 2 T_{i}},
$$

where $T_{i}$ is in $\mathrm{eV}$ and

$$
\begin{aligned}
n\left(\rho_{0}\right) & =\int f\left(\rho_{0}, v_{0}\right) d v^{3} \\
& =4 \pi \int_{0}^{\infty} v_{0}^{2} f\left(\rho_{0}, v_{0}\right) d v_{0},
\end{aligned}
$$

is chosen for simplicity.

The loss fraction is written as the following,

$$
\begin{aligned}
F & =2 \pi \oint \frac{d l}{B} \int_{-1}^{1} d \zeta_{0}\left[\int_{v_{T}}^{\infty}\left(\frac{m_{i}}{2 \pi k T_{i}}\right)^{3 / 2} v_{0}^{2} e^{-m_{i} v_{0}^{2} / 2 k T_{i}} d v_{0}+\right. \\
& \left.\int_{v_{B_{L}}}^{v_{B_{H}}}\left(\frac{m_{i}}{2 \pi k T_{i}}\right)^{3 / 2} v_{0}^{2} e^{-m_{i} v_{0}^{2} / 2 k T_{i}} d v_{0}\right] / \oint \frac{d l}{B} \\
& =\frac{1}{\sqrt{\pi}} \oint \frac{d l}{B} \int_{-1}^{1} d \zeta_{0}\left[\int_{E_{T}}^{\infty} E^{1 / 2} e^{-E} d E+\right. \\
& \left.\int_{E_{B_{L}}}^{E_{B_{H}}} E^{1 / 2} e^{-E} d E\right] / \oint \frac{d l}{B} \\
& =\frac{\oint \frac{d l}{B} \int_{-1}^{1}\left[\Gamma\left(3 / 2, E_{T}\right)+\Gamma\left(3 / 2, E_{B_{H}}\right)-\Gamma\left(3 / 2, E_{B_{L}}\right)\right] d \zeta_{0}}{\sqrt{\pi} \oint \frac{d l}{B}} .
\end{aligned}
$$

In the above, $\Gamma$ refers to the upper incomplete gamma function and a change of coordinates from velocities to kinetic energies was made.

We calculate various loss fractions for the L- and $\mathrm{H}-$ mode conditions for ASDEX Upgrade discharge 30628 and for ASDEX Upgrade H-mode shot 31533. As shown in Fig. 4 and Fig. 5, the primary relevant difference between the two H-mode discharges is the ion temperature profile. Disparities in the electric field and potential profiles are also important to keep in mind. Discharge 31533 has a deeper potential well with a minimum further in the plasma. While likely within experimental error bars, it is worth noting the effects on the theory. To further probe effects of the electric potential profile, we also consider both the experimental and neoclassically predicted fields for shot 30628 .

Fig. 12 shows the loss fractions for SOL sampling ions in all three scenarios while Fig. 13 shows the loss fractions for X-lost ions. Both cases demonstrate significant losses in the edge pedestal region of the plasma with the $\mathrm{X}$-losses being fewer and more localized to the edge. Both plots also include the loss fractions calculated in the absence of a radial electric field.

In general, the SOL sampling ion loss fractions are more significant for the H-mode plasmas than for the Lmode instance. However, in the near edge, the loss fraction is greater in the L-mode as a result of its less significant radial electric field; in the $\mathrm{H}$-mode cases, the radial (a)
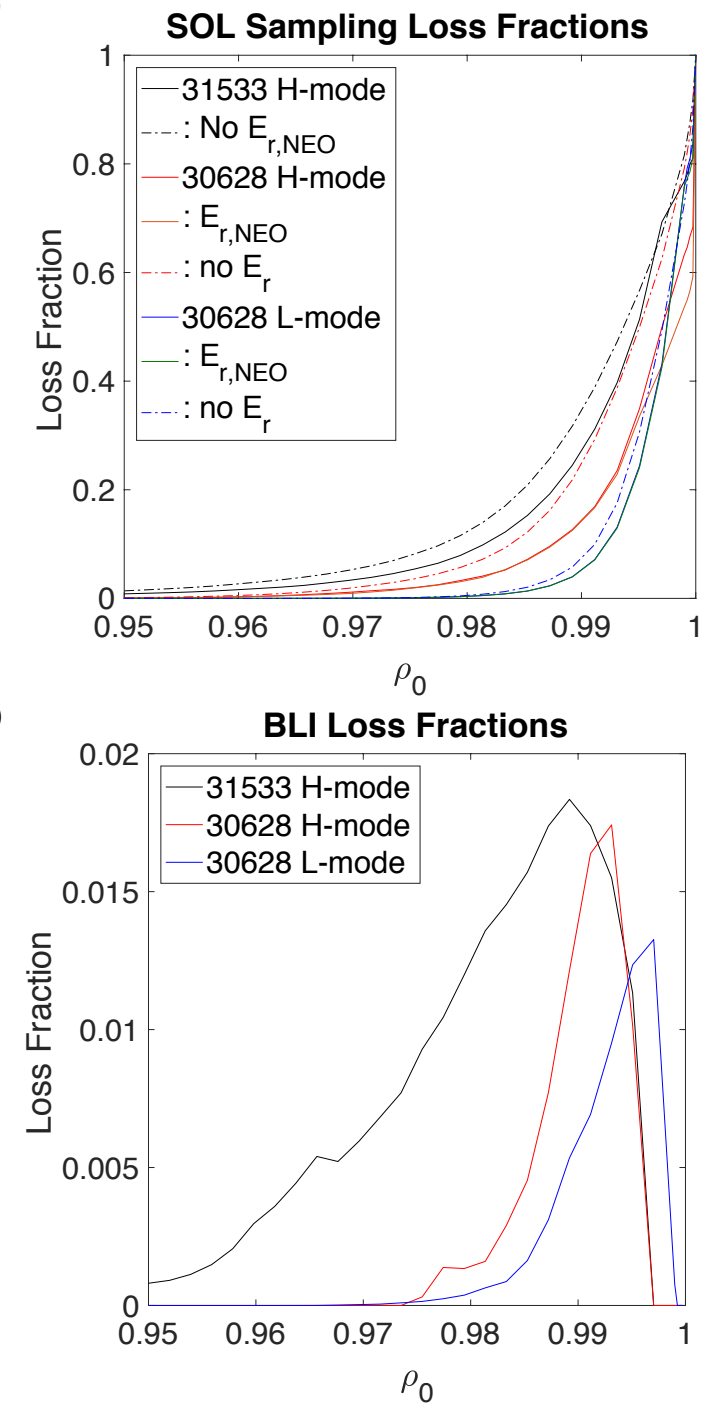

FIG. 12. Various loss fractions for SOL sampling ions. Plot (a) illustrates the total loss fractions for various accounts of the radial electric field while (b) shows the contributions from the banana orbit loss islands.

electric field is strong enough to taper off the growth of the loss fractions near the separatrix. Discharge 31533 exhibits a larger loss fraction than the $30628 \mathrm{H}$-mode primarily linked to its greater temperature profile. Disregarding the radial electric field provides simple curves for the loss fractions, which overestimate the number of ion losses. The starkest change when accounting for the effects of $E_{r}$ is seen in the H-mode loss fractions for shot 30628. It would appear that a higher ion temperature profile acts to mitigate the stronger effects of the electric field on ion orbit loss. This can also be inferred from Fig. 9. In the L-Mode, the temperature profile in the edge is sufficiently high that the raised velocity-space loss hole is still thermal. Conversely, in the H-mode the temperature profile is beneath the thresholds near the separatrix. For a similar radial electric field, a higher temperature profile would allow the higher threshold losses to be thermally 
(a)

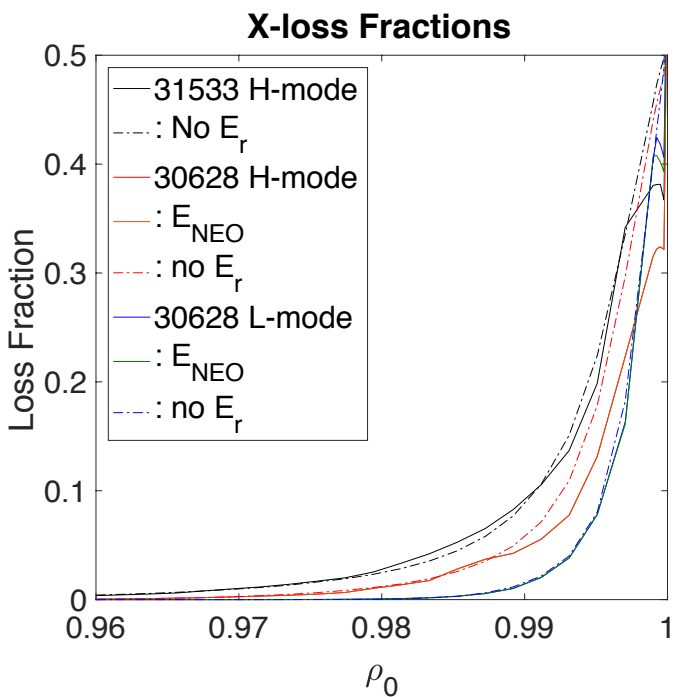

(b)

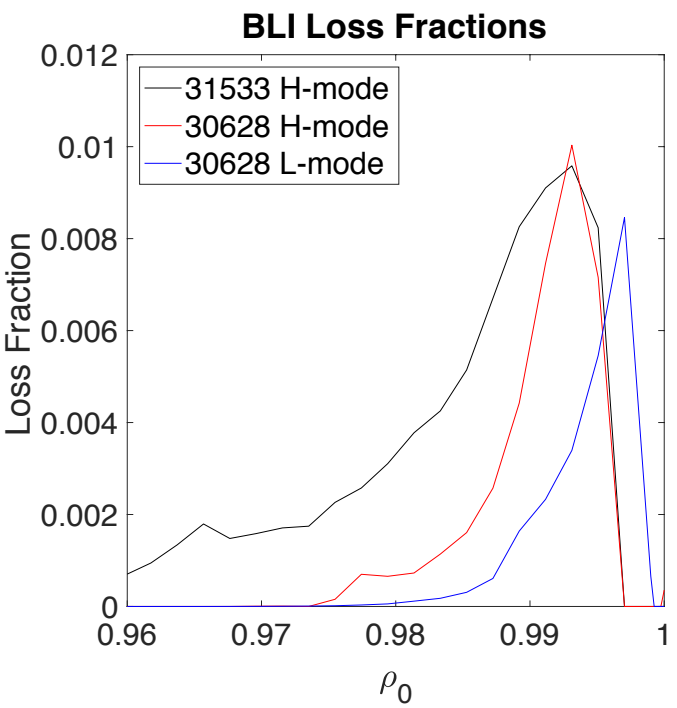

FIG. 13. The X-loss version of Fig. 11. Plot (a) includes the total loss fractions for different electric potential profiles and (b) shows the BLI loss fractions.

accessible.

There is a sizeable difference in the predicted losses for the H-mode instance of shot 30628 in the near edge, $\rho_{0}>0.995$, when using the neoclassical radial electric field in place of experimental measurements. This should have minimal consequences; the corresponding velocityspace loss hole is likely depopulated and the near edge has a relatively lower density of ions. Regardless, this is likely explainable by the second-derivative dissimilarities in the two potential profiles; the disparity begins near where the curvature differences are seen.

In the case of X-loss, shown in Fig. 13, similar behavior in the loss fractions can be seen. However, disregarding the electric field has a less than obvious effect on the X-loss loss fractions. $E_{r}$ growth evokes a competition between the overall shift in energy of the losses and the increased accessibility for trapped orbit losses in the plasma. As seen in Fig. 10, the most thermally accessible $\mathrm{X}$-losses are trapped orbits. Extremely near the edge, the effect of a strong electric field acts to reduce the X-losses, while it can be seen to enhance, or at least not diminish, the X-losses farther in the plasma due to the importance of banana orbit accessibility. This is seen particularly well in the higher ion temperature case of shot 31533, most notably between $\rho_{0} \sim 0.98$ and $\rho_{0} \sim 0.99$.

For both loss definitions, the BLI loss fractions behave similarly to each other. For the L-mode, the losses are least significant, but not negligible, and are most localized to the edge. Under H-mode conditions, the losses peak at just under $2 \%$ and $1 \%$, for SOL sampling and Xloss respectively, and decline to less than $0.1 \%$ near the respective pedestal tops, seen in the temperature profiles in Fig. 4. The loss fractions are also insignificant very near the separatrix; here, the BLIs have opened up to the rest of the velocity-space loss hole.

So far, our findings only apply to single instances in time. Following the ion orbit losses, the loss hole depopulates and the radial electric field strengthens, or the loss hole was already depopulated and only a diminished fraction of ions are lost. We highlight a few effects that are instrumental in treating the losses dynamically.

\section{Ion Orbit Loss as a Dynamic Effect}

As an inherently non-ambipolar transport mechanism, ion orbit loss is both sensitive to and is a source of the radial electric field via the resultant return current. Following the depopulation of the velocity-space loss hole, the radial electric field grows and the loss hole is sensitive to this change. Furthermore, the ion losses should be reflected in the time-evolved temperature and density profiles.

We will discuss a few aspects which find importance when considering ion orbit loss dynamically. The arguments are at most semi-quantitative, anything more is currently beyond the scope of the paper. The first concerns the return of 'lost' ions back into the main plasma. The second considers the repopulation of the velocityspace loss hole and the particular importance of the banana loss islands.

\section{A. Returning lons}

Our first definition of a lost orbit simply considered ions with SOL sampling orbits. In reality, some of these ions still follow closed orbits and many others may backscatter into the plasma while in the SOL. The true loss fraction,

$$
\tilde{F}=C F_{\mathrm{SOL}}, \quad 0<C \leq 1,
$$

should be the calculated SOL sampling loss fraction modified by a coefficient $C$, where $C$ is the fraction of particles that cross the LCFS and do not re-enter the plasma. 
Such a number is only a simple model as the likelihood of a SOL sampling ion re-entering the plasma would be highly dependent on the location it crosses the separatrix.

We now turn our attention to the X-lost ions. Ions lost near the $\mathrm{X}$-point have short paths to the divertor, and are considered less likely to re-enter the plasma. The ions are only lost near the X-point in the favorable forward $\nabla B$ configuration, otherwise they would be lost across the separatrix at the opposite end of the plasma, thus being maximally exposed to the return effects.

The loss fraction calculated following the first loss definition can be expressed as the sum of the loss fraction of ions lost via the X-Point and those lost anywhere else,

$$
F_{\mathrm{SOL}}=F_{X}+F_{\mathrm{Non}-X}
$$

where $F_{X}$ are the X-lost ions. We represent the true loss fraction, $\tilde{F}$, as the sum of these two parts where each is modified by a different loss coefficient

$$
\tilde{F}=C_{1} F_{X}+C_{2} F_{\text {Non- } X}, \quad 0<C_{2}<C_{1} \leq 1,
$$

where $C_{1}$ is expected to be much closer to unity.

By integrating the curves in Fig. 12 and Fig. 13, the fraction of the losses captured by our first loss definition that also meet our requirement for X-loss is found. Around $44.1 \%$ of SOL sampling ions in the L-mode and $40.2 \%$ in the H-mode for discharge 30628 and $39.7 \%$ for discharge 31533 are lost in this fashion. We take $F_{X} \approx 0.4 F_{\mathrm{SOL}}$, insinuating that if the tokamak is designed with drifts pointing towards the X-point, $40 \%$ of the SOL sampling ions would experience mitigated plasma re-entry. If the drifts are directed unfavorably, then the $40 \%$ of ion losses that would have been X-lost are maximally impacted by the SOL effects. In this case, the loss coefficient $C_{2}$ is simply applied to all losses. In the preferred drift direction

$$
\begin{aligned}
\tilde{F}_{\nabla B \downarrow} & =C_{1} F_{X}+C_{2} F_{\text {Non- } X} \\
& \simeq\left(\frac{4}{10} C_{1}+\frac{6}{10} C_{2}\right) F_{\mathrm{SOL}} .
\end{aligned}
$$

In the unpreferred drift direction

$$
\begin{aligned}
\tilde{F}_{\nabla B \uparrow} & =C_{2}\left(F_{X}+F_{\text {Non- } X}\right) \\
& =C_{2} F_{\mathrm{SOL}},
\end{aligned}
$$

which results in

$$
\frac{\tilde{F}_{\nabla B \downarrow}}{\tilde{F}_{\nabla B \uparrow}} \simeq \frac{1}{5}\left(2 \frac{C_{1}}{C_{2}}+3\right) .
$$

An orbit following study has shown that $80 \%$ of ions lost across the separatrix at a poloidal angle very near the $\mathrm{X}$-point will not re-enter the plasma while only around $40 \%$ of the ions that cross the LCFS elsewhere will reach the wall ${ }^{9}$. Respectively, using the above values for $C_{1}$ and $C_{2}$ indicates that the true loss fraction in the forward configuration is 1.44 times larger than that in the backward configuration. Another work indicates that of the $60 \%$ of non-X-lost ions with wall intersecting orbits, some fraction will re-enter the plasma via SOL effects. They take the adjusted loss coefficient to be $50 \%{ }^{7}$. This value for $C_{2}$ results in ion orbit loss being 1.24 times larger in the forward configuration. Assuming a linear relationship between the number of lost ions and radial electric field growth finds semi-quantitative agreement with simulation results where it was found that the downwards drift configuration features an electric potential well about $30 \%$ larger compared to the upwards drift $\operatorname{case}^{36}$.

Treating ion orbit loss dynamically requires appropriate classification of losses. SOL sampling ions and X-lost ions appear to be a good combination of definitions since the first captures all possible losses and the second captures losses with a known specific behavior. Continued studies for the recycling rates, and their parameter dependencies, for both types of ion orbit losses is instrumental for time evolution.

\section{B. Loss Hole Repopulation}

For the purposes of this discussion, let us again assume that the velocity-space particle distribution can be thought of as a Maxwellian about the local ion temperature. After the ion orbit losses, the Maxwellian will be truncated above the calculated threshold ion loss energies. As the distribution relaxes around a new temperature, previously accessible loss energies may no longer be thermally accessible and the loss hole will be empty. To successfully treat ion orbit loss as a progressive phenomenon, the mechanisms that repopulate the emptied regions of velocity-space must communicate well with the ion orbit loss model.

However, with the inclusion of the BLIs, not only is the Maxwellian truncated above the threshold loss energies, but also an additional slice of the Maxwellian is lost. Velocity-space both above and below the BLIs can be populated and under these conditions the banana loss islands will surely repopulate under relaxation. With an increase of $E_{r}$ magnitude, there is the potential for both the formation of new BLIs or the growth of existing ones. As mentioned towards the end of Section V.A.1., if the radial electric field grows sufficiently large in magnitude, the BLIs are prone to open and connect to the higher energy regions of velocity-space corresponding to losses on $\psi_{-}$. The BLIs are able to bleed out thermal ions more or less continuously until there is a strong enough local radial electric field or the Maxwellian has relaxed to a low enough energy to inhibit the losses.

\section{Conclusion}

We have developed a method to study the nonambipolar ion orbit loss in the axi-symmetric magnetic 
field of a tokamak by considering the intersection of an ion's constraint surface, $\psi_{ \pm}$, and the poloidal flux, $\Psi_{\text {pol }}$, determined by the Grad-Schlüter-Shafranov equation. The effects of the radial electric field are accounted for by determining which initial ion locations have accessible banana orbits for varying pitch direction. A full characterization of ion orbit loss includes not only threshold loss energies, upwardly unbounded loss regions, but also energy ranges which comprise banana loss islands, upwardly bounded loss regions.

Thermal ion losses are substantial in the pedestal region for two definitions of ion orbit loss: SOL sampling ions and X-lost ions. Many SOL sampling orbits are prone to re-enter the plasma which causes the first definition to over-estimate the number of losses. The second definition only considers the ions directly lost to the divertor very near the $\mathrm{X}$-point. The loss hole is shown to be more substantial in ASDEX Upgrade discharge 30628 in the H-mode than in the L-mode just prior to the L-H transition. The loss hole is further shown to be larger for a higher ion temperature $\mathrm{H}$-mode plasma, ASDEX Upgrade discharge 31533.

Additionally, we discussed a few key details that are important in determining the cumulative effects of ion losses as a function of time. First are the effects of lost ions returning to the plasma. The fraction of lost ions that are largely exempt from this phenomenon in the preferred $\nabla B$ drift direction is determined using the relationship between the two loss definitions. By comparing the loss fractions in both orientations of the $\nabla B$ drift, under realistic expectations for both SOL sampling and X-lost ions re-entering the plasma, there is a semiquantitative agreement with expectations of the ratio of $E_{r}$ generation in the forwards and backwards drifts, further highlighting the importance of considering both definitions of ion orbit loss. Second is the repopulation of the loss hole. BLIs are a means to bleed out the edge plasma since the loss holes are prone to thermally refilling. They provide a continuous, yet slight, source of ion loss while the radial electric field is strengthened. Eventually, the islands can open in the presence of a sufficiently large radial electric field and join the rest of the loss hole. For the $\mathrm{H}$-mode discharges, BLI losses diminish at the pedestal top.

To further develop the model as a tool to calculate the contribution of ion orbit loss to the radial electric field, several things must be considered. Statically, the model would be improved by taking into account the toroidal asymmetries in the equilibria or poloidal asymmetries in the plasma profiles. Furthermore, investigating the ion orbit loss dependence on X-point geometry would be insightful. Dynamically, the repopulation of the loss hole via pitch-angle scattering and other neoclassical effects is crucial in understanding how ion orbit loss can generate a radial electric field over a timescale longer than a typical toroidal bounce.

\section{Acknowledgments}

We would first like to give thanks to Antti Snicker and the developers of ASCOT $4^{37}$ for allowing use of their Monte Carlo orbit-following code. The visualization of the orbits was influential to the development of the ion orbit loss model.

We thank Mike Dunne in particular for providing the experimental profiles for discharge 31533.

Finally, we thank Alex Chankin, David Coster, Eleonora Viezzer, and Cole Stephens for conversations regarding the project. All provided clarity and direction.

This work has been carried out within the framework of the EUROfusion Consortium and has received funding from the Euratom research and training programme 2014-2018 under grant agreement No 633053. The views and opinions expressed herein do not necessarily reflect those of the European Commission.

\section{Appendix}

\section{A. Approximation of $p_{\phi}$}

Eq. (3) is an expression for the guiding center canonical momentum. Let us here first write the exact expression for the canonical momentum,

$$
p_{\phi}=m R v_{\phi}+e \psi,
$$

where all quantities are evaluated at the location of the ion. approximated $v_{\phi} \simeq v_{\|} \frac{B_{\phi}}{B}$. Here, we consider the validity of the approximation. We start from the definition of $v_{\|}$,

$$
\begin{aligned}
v_{\|} B & =\vec{v} \cdot \vec{B}=v_{\phi} B_{\phi}+v_{R} B_{R}+v_{z} B_{z} \\
v_{\|} & = \pm \sqrt{\frac{\left(v_{\phi} B_{\phi}+v_{R} B_{R}+v_{z} B_{z}\right)^{2}}{B^{2}}} \\
& = \pm v_{\phi} \sqrt{\frac{\left(1+\frac{v_{R} B_{R}}{v_{\phi} B_{\phi}}+\frac{v_{z} B_{z}}{v_{\phi} B_{\phi}}\right)^{2}}{1+\frac{B_{R}^{2}}{B_{\phi}^{2}}+\frac{B_{z}^{2}}{B_{\phi}^{2}}}},
\end{aligned}
$$

and make use of the neoclassical nature of the problem.

A direct connection between the magnetic field geometry ratios and the dynamics of the particle, the velocities, is made. To lowest order, the guiding center's trajectory follows magnetic field lines. The dominant field line in the tokamak is $B_{\phi}$. Thus,

$$
\frac{v_{R}}{v_{\phi}} \sim \frac{B_{R}}{B_{\phi}}, \quad \frac{v_{z}}{v_{\phi}} \sim \frac{B_{z}}{B_{\phi}} .
$$

In fact, a correction should be made for the guiding center drifts, $v_{D}$, which are the combination of both the $\nabla B$ and curvature drifts,

$$
\frac{v_{z}-v_{D}}{v_{\phi}} \sim \frac{B_{z}}{B_{\phi}}
$$


where,

$$
\begin{aligned}
\vec{v}_{D} & =\vec{v}_{\nabla B}+\vec{v}_{R_{c}}, \\
\vec{v}_{\nabla B} & =\frac{m v_{\perp}^{2}}{2 q B} \frac{\vec{B} \times \nabla B}{B^{2}}, \\
\vec{v}_{R_{c}} & =\frac{m v_{\|}^{2}}{q B} \frac{\vec{R}_{c} \times \vec{B}}{B R_{c}^{2}} .
\end{aligned}
$$

We continue with our expression for the parallel velocity,

$$
\begin{aligned}
v_{\|} & = \pm v_{\phi} \sqrt{\frac{\left(1+\frac{B_{R}^{2}}{B_{\phi}^{2}}+\frac{B_{z}^{2}}{B_{\phi}^{2}}+\frac{v_{D} B_{z}}{v_{\phi} B_{\phi}}\right)^{2}}{1+\frac{B_{R}^{2}}{B_{\phi}^{2}}+\frac{B_{z}^{2}}{B_{\phi}^{2}}}} \\
& = \pm v_{\phi} \frac{B}{B_{\phi}} \sqrt{1+\frac{v_{D}^{2} B_{\phi}^{2} B_{z}^{2}}{v_{\phi}^{2} B^{4}}+2 \frac{v_{D} B_{\phi} B_{z}}{v_{\phi} B^{2}}} .
\end{aligned}
$$

We have,

$$
\frac{B_{\phi}}{B} \leq 1, \quad \frac{B_{R}}{B} \leq \frac{B_{R}}{B_{\phi}} \ll 1, \quad \frac{B_{z}}{B} \leq \frac{B_{z}}{B_{\phi}} \ll 1
$$

Keeping only the highest order correction term shows that

$$
\begin{aligned}
v_{\|} & \simeq \pm v_{\phi} \frac{B}{B_{\phi}} \sqrt{1+2 \frac{v_{D}}{v_{\phi}} \frac{B_{z} B_{\phi}}{B^{2}}} \\
& \simeq \pm v_{\phi} \frac{B}{B_{\phi}}\left(1+\frac{v_{D}}{v_{\phi}} \frac{B_{z} B_{\phi}}{B^{2}}\right) .
\end{aligned}
$$

We simply solve for $v_{\phi}$,

$$
v_{\phi} \simeq v_{\|} \frac{B_{\phi}}{B}-\frac{B_{z} B_{\phi}}{B^{2}} v_{D}
$$

The positive sign was selected to ensure that $v_{\|}$is truly parallel to the magnetic field. The largest correction term is small and is appropriately dropped in the text.

\section{B. The Positiveness of $v_{0}$}

We will show that ensuring the positiveness of the bracketed terms in (8) and (9) is identical to ensuring that $v_{0}$ is positive.

This is easily achieved by deriving the minimum initial velocity for an intersection of $\psi_{+}$with the separatrix, at a loss point, $\mathcal{P}$, characterized by a poloidal location $\left(R_{L}, z_{L}\right)$, in the case of $\vec{E}=\overrightarrow{0}$,

$$
v_{0 \min }=\frac{q}{m} \frac{\psi_{\text {sep }}-\psi_{0}}{R_{0} f_{\phi 0} \zeta_{0}+R_{L} f_{\phi L} \sqrt{1-\frac{B_{L}}{B_{0}}\left(1-\zeta_{0}^{2}\right)}}>0,
$$

and connecting that to (8) in the same case, $\vec{E}=\overrightarrow{0}$. We find that

$$
\begin{aligned}
E_{\min +}= & \frac{q^{2}}{2 m \xi^{2}}\left[\frac{R_{0}}{R^{2}} \frac{f_{\phi 0}}{f_{\phi}^{2}} \zeta_{0}\left(\psi_{\mathrm{sep}}-\psi_{0}\right)\right. \\
& \left.-\frac{\left(\psi_{\mathrm{sep}}-\psi_{0}\right)}{f_{\phi} R} \sqrt{R_{b}^{*}}\right]\left.^{2}\right|_{\mathcal{P}} \\
= & \frac{1}{2} m \tilde{v}_{0}^{2},
\end{aligned}
$$

which implies that

$$
\begin{aligned}
\tilde{v}_{0} & = \pm\left.\frac{q}{m R f_{\phi} \xi}\left(\psi_{\text {sep }}-\psi_{0}\right)\left[\frac{R_{0} f_{\phi 0}}{R f_{\phi}}-\sqrt{R_{b}^{*}}\right]\right|_{\mathcal{P}} \\
& = \pm\left.\frac{q}{m R f_{\phi}}\left(\psi_{\text {sep }}-\psi_{0}\right) \frac{\frac{R_{0} f_{\phi 0}}{R f_{\phi}}-\sqrt{R_{b}^{*}}}{\frac{R_{0}^{2}}{R^{2}} \frac{f_{\phi 0}^{2}}{f_{\phi}^{2}} \zeta_{0}^{2}-R_{b}^{*}}\right|_{\mathcal{P}} \\
& = \pm \frac{q}{m} \frac{\psi_{\text {sep }}-\psi_{0}}{R_{0} f_{\phi 0} \zeta_{0}+R_{L} f_{\phi L} \sqrt{1-\frac{B_{L}}{B_{0}}\left(1-\zeta_{0}^{2}\right)}} .
\end{aligned}
$$

We see $v_{0 \min }$ and $\tilde{v}_{0}$ are equivalent when the ' + ' sign is chosen. Therefore, keeping the bracketed terms in (8) and (9) positive, is the same as ensuring that $v_{0}$ is positive in order to be consistent with the definition of $p_{\phi}$.

${ }^{1}$ F. Wagner, G. Becker, K. Behringer, D. Campbell, A. Eberhagen, W. Engelhardt, G. Fussmann, O. Gehre, J. Gernhardt, G. v. Gierke, G. Haas, M. Huang, F. Karger, M. Keilhacker, O. Klüber, M. Kornherr, K. Lackner, G. Lisitano, G.G. Lister, H.M. Mayer, D. Meisel, E.R. Müuller, H. Murmann, H. Niedermeyer, W. Poschenrieder, H.Rapp, H. Röhr, F. Schneider, G. Siller, E. Speth, A. Stäbler, K.H. Steuer, G. Venus, O. Vollmer, and Z. Yü, Physical Review Letters 49, 19 (1982).

${ }^{2}$ R.J. Groebner, K.H. Burrell, and R.P. Seraydarian, Physical Review Letters 64, 25 (1990).

${ }^{3}$ V. Rozhansky and M. Tendler, Physics of Fluids B: Plasma Physics 4, 1877 (1992).

${ }^{4}$ F.L. Hinton and M.S. Chu, Nucl. Fusion 25, 345 (1985).

${ }^{5}$ F.L. Hinton, Nucl. Fusion 25, 1457 (1985).

${ }^{6}$ C.S. Chang, S. Ku, G.R. Tynan, R. Hager, R.M. Churchill, I. Cziegler, M. Greenwald, A.E. Hubbard, and J.W. Hughes, Physical Review Letters 118, 175001 (2017).

${ }^{7}$ W.M. Stacey, Nucl. Fusion 57, 066034 (2017).

${ }^{8}$ M. Rodriguez-Ramos, M. Garcia-Munoz, M.C. Jimenez-Ramos, J. Garcia Lopez, J. Galdon-Quiroga, L. Sanchis-Sanchez, J. Ayllon-Guerola, M. Faitsch, J. Gonzalez-Martin, A. Hermann, P. de Marne, J.F. Rivero-Rodriguez, B. Sieglin, A. Snicke, and the ASDEX Upgrade Team, Plasma Phys. Control. Fusion 59, 105009 (2017).

${ }^{9}$ T.M. Wilkes and W.M. Stacey, Physics of Plasmas 23, 102504 (2016).

10 J.S. Degrassie, J.A. Bodeo, and B.A. Grierson, Physics of Plasmas 22, 080701 (2015).

${ }^{11}$ A.V. Chankin and G.M. McCracken, Nucl. Fusion 33, 1459 (1993).

${ }^{12}$ K. Miyamoto, Nucl. Fusion 36, 927 (1996).

${ }^{13}$ J.A. Rome and Y-K.M. Peng, Nucl. Fusion 19, 1193 (1979).

${ }^{14}$ C.T. Hsu and D.J. Sigmar, Physics of Fluids B: Plasma Physics 4, 1492 (1992).

${ }^{15}$ S. Putvinskii, Plasma Physics Control. Fusion 35, 219 (1993).

${ }^{16}$ C.S. Chang, S. Kue, and H. Weitzner, Physics of Plasmas 9, 9 (2002).

${ }^{17}$ S. Ku, and C.S. Chang, Physics of Plasmas 11, 12 (2004). 
${ }^{18}$ D.J. Battaglia, C.S. Chang, S.M. Kaye, K. Kim, S. Ku, R. Maingi, R.E. Bell, A. Diallo, S. Gerhardt, B.P. LeBlanc, J. Menard, M. Podesta, and the NSTX Team, Nucl. Fusion 53, 113032 (2013).

${ }^{19}$ C. Bourdelle, L. Chôné, N. Fedorczak, X. Garbet, P.Beyer, J. Citrin, E. Delabie, G. Dif-Pradalier, G. Fuhr, A. Loarte, C.F. Maggi, F. Militello, Y. Sarazin, L. Vermare, and JET Contributors 073015 (2015)

${ }^{20}$ J.F. Chang, M. Isobe, K. Ogawa, J. Huang, C.R. Wu, Z. Xu, Z. Jin, S.Y. Lin, L.Q. Hu, and EAST Team, Review of Scientific Instruments 87, 11E728 (2016).

${ }^{21}$ W. Stacey, Physics of Plasmas 18, 102504 (2011).

${ }^{22}$ W.M. Stacey, Nucl. Fusion 53, 063011 (2013).

${ }^{23}$ J.S. deGrassie, R.J. Groebner, K.H. Burrell, and W.M. Solomon, Nucl. Fusion 49, 085020 (2009).

${ }^{24}$ J.S. deGrassie, S. H. Müller, and J.A. Boedo, Nucl. Fusion 52, 013010 (2012).

${ }^{25}$ W. Stacey, J.A. Boedo, T.E. Evans, B.A. Grierson, and R.J. Groebner, Physics of Plasmas 19, 112503 (2012).

${ }^{26}$ C. Pan, S. Wang, and J. Ou, Nucl. Fusion 54, 10 (2014).

${ }^{27}$ W.M. Stacey and B.A. Grierson, Nucl. Fusion 54, 073021 (2014).

${ }^{28}$ E. Viezzer, T. Pütterich, G.D. Conway, R. Dux, T. Happel, J.C. Fuchs, R.M. McDermott, F. Ryter, B. Sieglin, W. Suttrop, M. Willensdorfer, E. Wolfrum, and the ASDEX Upgrade Team, Nucl. Fusion 53, 053005 (2013).

${ }^{29}$ M. Cavedon, T. Pütterich, E. Viezzer, F.M. Laggner, A. Bur- ckhart, M. Dunne, R. Fischer, A. Lebschy, F. Mink, U. Stroth, M. Willensdorfer, E. Wolfrum, and the ASDEX Upgrade Team, Plasma Phys. Control. Fusion 59, 105007 (2017).

${ }^{30}$ T. G. Northrop and J. A. Rome, The Physics of Fluids 21, 384 (1978).

${ }^{31}$ C.D. Stephens, R.W. Brzozowski III, and F. Jenko, Physics of Plasmas 24, 102517 (2017).

${ }^{32}$ R. Maingi, C.S. Chang, S. Ku, T. Biewer, R. Maqueda, M. Bell, R. Bell, C. Bush, D. Gates, S. Kaye, H. Kugel, B. LeBlanc, J. Menard, D. Mueller, R. Raman, S. Sabbagh, V. Soukhanovskii, and the NSTX Team, Plasma Physics Control. Fusion 46, A305 (2004).

${ }^{33}$ V. Rozhansky, E. Kaveeva, P. Molchanov, I. Veselova, S. Voskoboynikov, D. Coster, A. Kirk, S. Lisgo, and E. Nardon, Nucl. Fusion 50, 034005 (2010).

${ }^{34}$ E. Viezzer, T. Pütterich, C. Angioni, A. Bergmann, R. Dux, E. Fable, R. M. McDermott, U. Stroth, E. Wolfrum and the ASDEX Upgrade Team, Nucl. Fusion 54, 012003 (2013).

${ }^{35}$ C.S. Chang and S. Ku, Physics of Plasmas 15, 062510 (2008).

${ }^{36}$ C.S. Chang, S.Ku, and H.Weitzner, Physics of Plasmas 11, 2649 (2002).

${ }^{37}$ E. Hirvijoki, O. Asunta, T. Koskela, T. Kurki-Suonio, J. Miettunen, S. Sipila, A. Snicker, S. Äkäslompolo, Computer Physics Communications 185, 1310-1321 (2014). 